\title{
Physical Activity Volumes during Pregnancy: A Systematic Review and Meta-Analysis of Observational Studies Assessing the Association with Infant's Birth Weight
}

\author{
Michèle Bisson, $\mathrm{MSc}^{1,2}$ Joëlle Lavoie-Guénette, $\mathrm{BSc}^{1}$ Angelo Tremblay, $\mathrm{PhD}^{2} \quad$ Isabelle Marc, MD, $\mathrm{PhD}^{1}$ \\ ${ }^{1}$ Department of Pediatrics, Centre Hospitalier Universitaire de Québec \\ (CHU de Québec), Université Laval, Quebec, Canada \\ 2 Department of Kinesiology, Faculty of Medicine, Université Laval, \\ Address for correspondence Isabelle Marc, MD, PhD, Department of \\ Pediatrics, CHU de Québec, 2705 Laurier Boulevard, Quebec, Canada \\ G1V 4G2 (e-mail: isabelle.marc@crchudequebec.ulaval.ca).
} Quebec, Canada

Am J Perinatol Rep 2016;6:e170-e197.

\begin{abstract}
Keywords

- birth weight

- body composition

- physical activity

- pregnancy

- systematic review

Objective This study aims to examine the association between different maternal physical activity exposures during pregnancy and infant's birth weight, body composition, and risk of inadequate weight.

Methods Two reviewers (M.B. and J.L.G.) identified observational studies reporting total or leisure time activity during pregnancy and birth weight outcomes. Pooled analyses were performed to summarize the risk associated with high or moderate volumes of physical activity on birth weight.

Results A total of 54 studies among 4,080 reported the association between physical activity and birth weight (37 studies) or risks of small or large birth weight. The association between physical activity and birth weight was evaluated by physical activity levels (low, moderate, or high). Despite heterogeneity, pooled results (23 studies) suggested that moderate levels of activity are associated with an increased birth weight (mean difference: $61.5 \mathrm{~g}, 95 \%$ confidence interval [Cl]: 16.6, 106.5, 15 studies), while high levels were associated with lower birth weight (mean difference: $-69.9 \mathrm{~g}, 95 \% \mathrm{Cl}$ : $-114.8,-25.0,15$ studies). Data were insufficient to provide robust estimates for other outcomes.

Conclusions The results of observational studies suggest an inverted u-shaped association between physical activity and birth weight, despite methodological variability. These results could help refining physical activity guidelines for pregnancy and provide guidance for future research.
\end{abstract}

As a marker of fetal growth, birth weight, and potentially neonatal body composition, is associated with health markers in later life such as body composition, ${ }^{1}$ strength, and cardiorespiratory fitness. ${ }^{2-4}$ Birth weight extremes are also associated with adverse health outcomes, including obesity, cardiovascular diseases, and metabolic dysfunction. ${ }^{5-8}$ Accordingly, these

received

January 16, 2016

accepted after revision

March 3, 2016
DOI http://dx.doi.org/

$10.1055 / \mathrm{s}-0036-1583169$. ISSN 2157-6998. outcomes are largely studied, although their determinants remain partly understood.

Leisure time physical activity is associated with the course of a healthy pregnancy, as it can increase cardiorespiratory fitness, ${ }^{9}$ decrease gestational weight gain, ${ }^{10}$ and may lower the risk of preeclampsia, ${ }^{11}$ cesarean section, ${ }^{12}$ and gestational
Copyright $\odot 2016$ by Thieme Medical Publishers, Inc., 333 Seventh Avenue, New York, NY 10001, USA. Tel: +1(212) 584-4662.
License terms

(이 (1) $\Theta \circledast$ 
diabetes. ${ }^{13,14}$ Recently, meta-analyses of randomized controlled trials (RCTs) have been published regarding the effect of maternal physical activity interventions on infant's birth weight, suggesting a modest yet significant decrease in birth weight with physical activity interventions. ${ }^{15,16}$

The important heterogeneity in physical activity interventions during pregnancy and adherence issues might nevertheless preclude a complete portrait of the impact of physical activity on markers of fetal growth, as this impact is likely to depend on the type, volume, and intensity of physical activity, as well as the conditioning level of the mother and timing during pregnancy. ${ }^{11,17-20}$ For instance, RCTs are usually initiated during the second trimester of pregnancy, making it difficult to assess the impact of exercise in early pregnancy.

Also, interventions are limited to moderate intensity physical activity, as it is the intensity recommended by numerous pregnancy guidelines. ${ }^{21,22}$ However, documenting the effects of vigorous intensity activities is crucial to ensure the safety of pregnant women who engage in such activities. In that regard, summarizing data from observational studies could greatly help understanding the effect of this somewhat prevalent behavior. Although previous meta-analyses of RCTs provided important evidence regarding the effect of maternal physical activity on birth weight, data from these studies are insufficient to determine whether these effects are specific to a particular volume, intensity, and timing of physical activity during pregnancy, and whether they rely on a dose-response relationship or if a threshold must be reached to achieve an effect.

Given the large number of observational studies and their potential to complement our understanding of the association between maternal physical activity and fetal growth, we performed a systematic review and when possible a metaanalysis of observational studies with the aim of characterizing the association between different maternal physical activity exposures (volume, intensity, timing during pregnancy, and measurement method) and infant birth weight, body composition, and risk of inappropriate birth weight for gestational age.

\section{Methods}

\section{Sources}

The protocol was published online on the international prospective register of systematic reviews Web site (CRD42013004968). The search for citations in English or French was performed in PubMed, EMBASE, and Web of Science, considering all articles published from 1946, 1947, and 1956 depending on the database, until June 2013. A second search considering articles published between June 2013 and March 2015 was subsequently performed. Search in PubMed included the following MeSH and/or text terms for physical activity: "exercise," "exertion," "pliability," "physical fitness," "physical education and training," "exercising," "physical condition," "stamina," "sports," and "muscle strengthening exercises." For weight outcomes, the following MeSH or/and text terms were used: "pregnancy," "pregnancy complications," "pregnancy outcome," "birth weight," "low birth weight," "high birth weight," "small for gestational age," "large for gestational age," "pregnancy," "pregnanc*," "pregnant"," "pregnancy complication*," "pregnancy outcome," "birth weight," "low birth weight," "high birth weight," "small for gestational age," and "large for gestational age." Terms were combined using the Boolean operator "OR" within each category, and "AND" when combining between exposure and outcome. A similar approach was used with other databases.

\section{Study Selection}

Studies were excluded if they were conference abstracts, reviews, case reports, unpublished articles, or intervention trials. Reviews were manually searched for citations not detected by the electronic search. Citations were combined and duplicates excluded. As prespecified in the protocol, studies were considered only if they measured leisure time or total physical activity. Two reviewers (M. B., J. L. G.) independently screened titles for potential relevance. Titles obviously not related to the topic of interest were excluded. Potentially relevant abstracts were screened using a standardized criterion (i.e., human studies, reference to pregnancy or birth weight or physical activity). Abstracts considered by both reviewers as not relevant were excluded, and full articles were retrieved. Any disagreement was solved by discussion and consensus between the two reviewers ( $M$. B., J. L. G.) and a third author (I. M.) if needed. All included articles were independently reviewed for data extraction on a form, especially conceived before extraction, as follows: study year and country, population, design, the number of exposed/ unexposed or cases/controls, definitions, and methods for exposure assessment, including type, frequency, intensity, and timing of physical activity, definitions, and methods for outcome assessment, effect estimates or continuous data, and adjustment variables. Studies were classified based on the design and by the outcome. Study quality was assessed using the Newcastle-Ottawa quality assessment scale. ${ }^{23}$

\section{Outcomes}

The outcomes of interest were the neonate's birth weight in grams, low and high birth weight (LBW, HBW), defined as a birth weight below 2,500 $\mathrm{g}$ and above 4,000 g, respectively, small and large for gestational age (SGA, LGA) if birth weight for gestational age was below the 10th and above the 90th percentile, respectively, and body composition, defined as fat percentage, fat mass or lean mass in grams.

\section{Exposure}

Maternal physical activity was defined as either leisure time physical activity or total physical activity performed during pregnancy and not related to an intervention. Leisure time activity (including sports, exercise, and recreational activities) was chosen as it is the focus of physical activity recommendations. However, since leisure time activity only accounts for around $10 \%$ of pregnant women's energy expenditure, ${ }^{24,25}$ total activity (including all domains, e.g., workrelated activity) was also considered. To be as inclusive as possible, it was determined that physical activity could be defined according to volume (product of frequency, intensity, 
and duration), frequency, intensity, and/or duration alone. With the increasing popularity of objective measurement devices (e.g., accelerometers), we also wanted to verify whether studies using such technology, or other objective methods to measure physical activity, would provide more consistent results compared with the overall evaluation of all eligible studies.

To investigate whether the association between physical activity and birth weight outcomes differed by physical activity levels, study groups (when available) were classified as "high level," "moderate level," or "low level/sedentary." A study group was classified as "high level" if the authors stated that this group performed vigorous intensity activity, if the group performed a high volume of activity, defined as at least three sessions per week of at least 30 minutes (which is similar to the recommendations of vigorous activity for the general population $)^{26}$ and ideally with a specification of intensity, or if the group was the one with the highest energy expenditure, duration, or intensity of physical activity in studies with more than two groups, allowing the comparison of groups with the largest difference in physical activity levels. Therefore, in this review, "level" refers to physical activity categorization regardless of whether a study defines physical activity by volume, frequency, intensity, or duration. Study groups not meeting the criteria of "high level" of activity or classified as "moderately active" by the authors were included in our "moderate level" comparison. In both the cases, moderate- and high-level groups were compared with the group having the lowest activity level in the study.

\section{Data Analysis}

Due to an important heterogeneity in the way studies defined, measured and quantified physical activity, studies were exclusively pooled to answer our main question regarding how physical activity volume and/or intensity could modulate infant's birth weight, based on a cautious classification of studies (as described above). To account for variability across studies and to derive conservative assessments of the uncertainty in the estimates, a Der Simonian and Laird random effects model was used. ${ }^{27}$ Data were pooled using the weighted unstandardized mean difference in effect size across studies. Heterogeneity was assessed with the $I^{2}$ statistic, ${ }^{28}$ with high heterogeneity defined as $I^{2} \geq 75 \%{ }^{28}$ The results were considered significant if $p<0.05$.

\section{Results}

\section{Characteristics of the Studies}

Among the 4,080 identified citations, 54 studies met the inclusion criteria (-Fig. 1). There were 50 cohort studies and 4 case-control studies. ${ }^{29-32}$ Overall, the studies were of moderate (28 studies, $51.9 \%)^{29,31-57}$ or good (21 studies, $38.9 \%)^{30,58-77}$ methodological quality, while 5 studies (9.2\%) were classified as of poor methodological quality. ${ }^{78-82}$ Most studies were conducted in a community setting (59.3\%) and included low-risk, predominantly Caucasian women. Characteristics of the studies are summarized in - Table $\mathbf{1}$, while detailed descriptions of exposures and outcomes of individual studies can be found in - Table 2 .

Physical activity was mainly assessed with questionnaires (including interviews and diaries), but only 10 studies used a questionnaire specifically validated for pregnancy. $^{31,32,43,44,59,63,67,72,75,76}$ Leisure time activity was more often reported than total physical activity. Only eight studies included objective physical activity measures (heart rate monitoring, ${ }^{36,37,48}$ use of pedometers, ${ }^{46}$ or accelerometers ${ }^{41,45,48,73,77}$ ). Vigorous activity was reported in eight studies, ${ }^{33,36,37,40,53,57,67,71}$ with the most common definition as follows: exercise at or above $50 \%$ of maximal capacity. $33,36,37,57$

A total of 15 studies (27.8\%) reported physical activity as a summary measure for the entire pregnancy. $32,34-37,42,47,49,54,56,57,60,68,78,81$ Numerous studies reported results for more than one trimester, but only eight studies (14.8\%) assessed physical activity in each trimester of pregnancy. ${ }^{30,50,51,58,59,61,64,75}$ Physical activity was treated as a categorical variable in most studies. However, the number of physical activity groups varied, as well as the criteria used to define these groups. Indeed, some studies compared active women to a group of women not doing any leisure time activity, while other studies used a "low activity" comparison group or created groups of various physical activity levels (using the median, tertiles, quartiles, etc.).

Most studies extracted birth weight outcomes from medical charts. Body composition was derived from the skinfold measure in one study, ${ }^{36}$ whereas it was evaluated by airdisplacement plethysmography in another one. ${ }^{75}$ Most studies defined birth weight outcomes as prespecified in the protocol, with a few exceptions using close definitions. ${ }^{38,49,51,53,55,61,65,72,73,79,81}$

A total of 34 studies adjusted for confounding variables, mainly maternal weight or body mass index, gestational weight gain, smoking, age, parity, education, gestational age at delivery, and infant sex. However, the choice of confounders varied substantially between studies.

\section{Association between Maternal Physical Activity and Birth Weight}

\section{Global Results of the Association between Maternal Physical Activity and Birth Weight}

A total of 37 studies (68.5\% of all 54 studies) reported the association between physical activity (total and/or leisure time physical activity) and birth weight. Among these studies, 25 (67.6\%) did not observe a statistically significant difference in birth weight within various physical activity levels, or vice versa. Conversely, 8 of the 37 studies (21.6\%) found a significant decrease in birth weight with increasing maternal activity, ${ }^{33,36,48,57,62,67,68,75}$ while physical activity was associated with an increased birth weight in the last 4 studies (10.8\%). ${ }^{38,44,64,79}$ A total of 21 studies adjusted for confounders, with similar results; 14 (66.7\%) did not find a significant association between maternal physical activity and birth weight, while 5 (23.8\%) found a significant decrease, ${ }^{33,62,67,68,75}$ and 2 


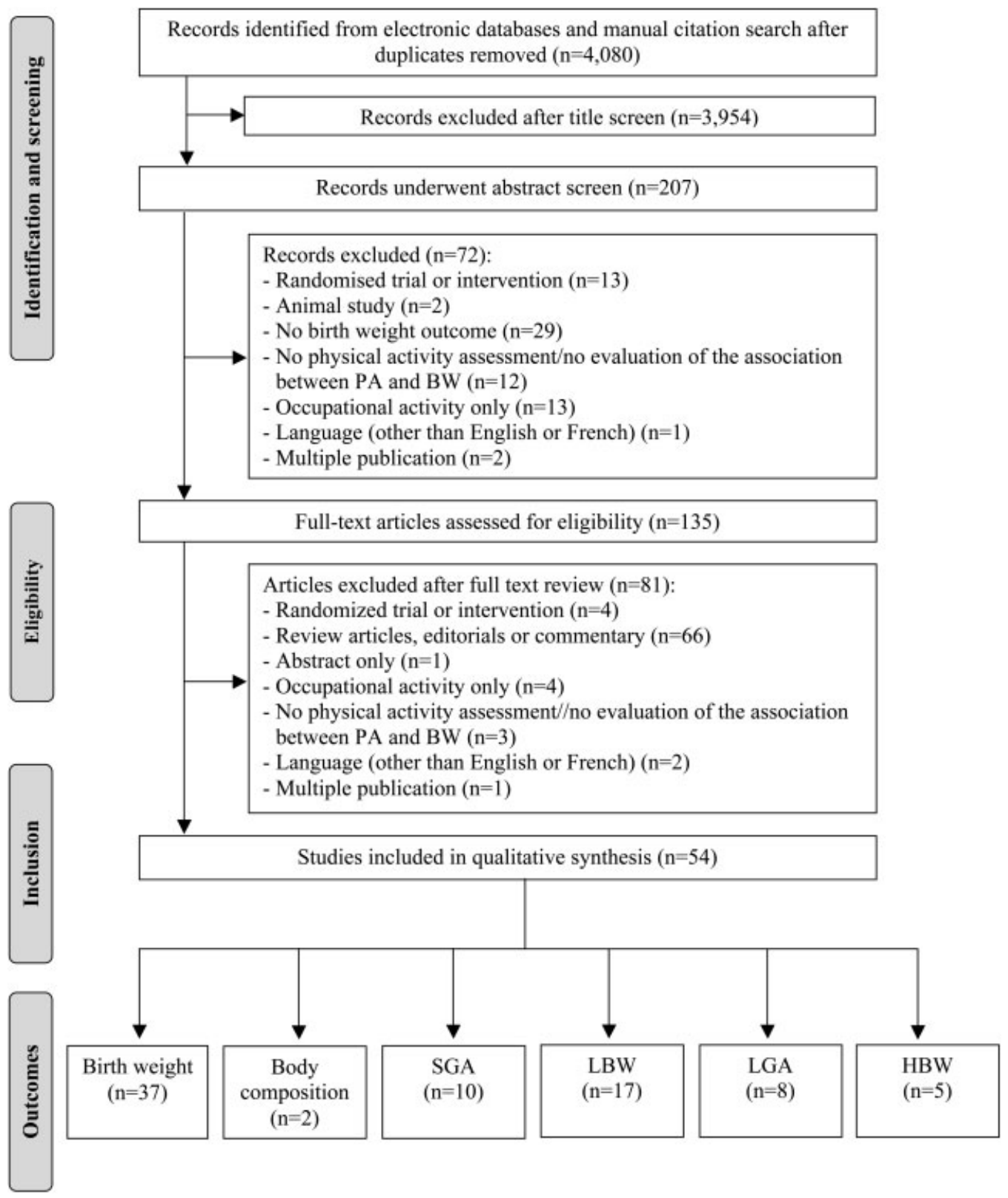

Fig. 1 Flowchart of reviewed citations. HBW, high birth weight; LBW, low birth weight; LGA, large for gestational age; SGA, small for gestational age.

(9.5\%) found a significant increase in birth weight with increasing activity. ${ }^{44,64}$

Among studies that objectively measured physical activity and looked at the association with birth weight, two found a significant decrease in birth weight with increasing activi$\mathrm{ty}^{36,48}$ while the remaining five studies found no significant association birth weight. ${ }^{37,41,45,46,73}$

Birth Weight Results According to Physical Activity Levels (High or Moderate vs. Low Levels)

Data from 23 studies (62.2\% of the 37 studies) could be pooled to evaluate the association between physical activity levels and birth weight. Overall, 17 studies (45.9\% of the 37 studies) reported the association between physical activity during pregnancy and birth weight comparing high levels of physical activity with no activity or low levels (-Fig. 2). ${ }^{33,36,37,40,48,50,53,56,57,64-68,71,74,75}$ Two of them (reporting no significant association between physical activity and birth weight) could not be pooled as they did not report enough quantitative data. ${ }^{40,53}$ From the remaining 15 studies, most (13) reported leisure time physical activity and 9 presented adjusted estimates. ${ }^{50,64-68,71,74,75}$ Despite the significant heterogeneity, individual results and pooled estimates of these studies suggested a decrease in birth weight with high levels of physical activity during pregnancy, compared with low levels. Considering only studies evaluating leisure time physical activity did not change these results (mean difference: $-56.1 \mathrm{~g}, 95 \%$ confidence interval $[\mathrm{CI}]:-100.5,-11.8$, $n=13$ studies). 
Table 1 Studies' characteristics

\begin{tabular}{|c|c|}
\hline Characteristics & $n(\%)$ \\
\hline \multicolumn{2}{|l|}{ Study design } \\
\hline Cohort & $50(92.6)$ \\
\hline Case-control & $4(7.4)$ \\
\hline \multicolumn{2}{|l|}{ Year of publication } \\
\hline Since 2010 (past 5 y) & $21(38.9)$ \\
\hline \multicolumn{2}{|l|}{ Origin of the studies } \\
\hline North America & $29(53.7)$ \\
\hline Canada & $3(5.6)$ \\
\hline Europe & $14(25.9)$ \\
\hline Oceania & $4(7.4)$ \\
\hline Asia & $4(7.4)$ \\
\hline South America & $3(5.6)$ \\
\hline \multicolumn{2}{|l|}{ Setting } \\
\hline Community-based & $32(59.3)$ \\
\hline Hospital-based & $21(38.9)$ \\
\hline Military-based & $1(1.9)$ \\
\hline \multicolumn{2}{|l|}{ Sample size } \\
\hline$<100$ & $12(22.2)$ \\
\hline $100-<1,000$ & $25(46.3)$ \\
\hline $1,000-10,000$ & $12(22.2)$ \\
\hline$\geq 10,000$ & $5(9.3)$ \\
\hline \multicolumn{2}{|l|}{ Ethnicity } \\
\hline Caucasians or mixed ethnicities & $47(87.0)$ \\
\hline Other $^{\mathrm{a}}$ & $7(13.0)$ \\
\hline \multicolumn{2}{|l|}{ Physical activity exposure } \\
\hline Leisure time physical activity & $41(75.9)$ \\
\hline Total activity & $19(35.2)$ \\
\hline \multicolumn{2}{|l|}{ Physical activity assessment ${ }^{\mathrm{b}}$} \\
\hline Self-report & $49(90.7)$ \\
\hline Objective measure & $8(14.8)$ \\
\hline \multicolumn{2}{|l|}{ Time covered by physical activity assessment } \\
\hline Whole pregnancy & $15(27.8)$ \\
\hline First trimester & $10(18.5)$ \\
\hline Second trimester & $20(37.0)$ \\
\hline Third trimester & $23(42.6)$ \\
\hline Each trimester separately & $8(14.8)$ \\
\hline Variable/unspecified & $3(5.6)$ \\
\hline \multicolumn{2}{|l|}{ Birth weight outcomes } \\
\hline Birth weight & $37(68.5)$ \\
\hline Body composition & $2(3.7)$ \\
\hline SGA/LBW & $27(50.0)$ \\
\hline LGA/HBW & $13(24.1)$ \\
\hline \multicolumn{2}{|l|}{ Outcome provenance } \\
\hline Medical charts or measured by study team & $39(72.2)$ \\
\hline Maternal recall & $6(11.1)$ \\
\hline Unspecified & $9(16.7)$ \\
\hline
\end{tabular}

Abbreviations: HBW, high birth weight; LBW, low birth weight; LGA, large for gestational age; SGA, small for gestational age.

${ }^{a}$ Two studies conducted in African Americans, two in Asians, two in West Asians, and one in Hispanic women.

'Three studies used both objective and subjective methods.
Similarly, looking at the difference in infant's birth weight from the 17 studies $^{34,37-40,45,50,53,55,57,65-67,71,73,79,80}$ comparing moderate levels of physical activity with low or no activity during pregnancy suggested that moderate levels increased birth weight ( - Fig. 3). Considering only studies assessing leisure time physical activity, reported in 12 of the 15 pooled studies ( 2 studies reporting nonsignificant results could not be combined due to insufficient quantitative data), ${ }^{40,53}$ did not change these findings (mean difference: 74.2 g, 95\% CI: 24.9, 123.5, $n=12$ studies).

\section{Birth Weight Results According to Leisure Time or Total Physical Activity}

A total of 25 studies ( $67.6 \%$ of the 37 studies) investigated the association between leisure time physical activity and birth weight. Five found a significant decrease in birth weight with increasing activity, ${ }^{33,36,57,62,67} 3$ found a significant increase in birth weight with increasing activity ${ }^{38,64,79}$ while the remaining 17 found no association. $34,37,39,43,50,53,54,56,59,65,66,71,74,78,80-82 \quad$ Total physical activity was evaluated in 13 studies (35.1\%), with 3 studies finding a significant decreased birth weight with increasing activity, ${ }^{48,68,75} 1$ study observing the contrary ${ }^{44}$ and the other 9 studies finding no significant association. ${ }^{40,41,45,46,52,55,68,72,73}$

\section{Birth Weight Results According to Physical Activity Timing during Pregnancy}

The majority of studies evaluating first trimester physical activity did not observe any significant association with birth weight, ${ }^{50,55,59,64,75}$ while one found a decreased birth weight within women doing a leisure time activity. ${ }^{67}$ Similar results were observed for the second trimester; most studies found no significant association with birth weight. ${ }^{41,50,52,59,65,71,74,75}$ Two studies observed a decreased birth weight with increasing leisure time activity ${ }^{33,62}$ while two others found the opposite with either total ${ }^{44}$ or leisure time activity. ${ }^{64}$ Again, for the third trimester, most studies did not observe an association between maternal activity and birth weight. ${ }^{41,45,50,52,59,64,65,80,82}$ Two studies found a decreased birth weight with increasing total ${ }^{75}$ or leisure time activity $^{62}$ and one found an increased birth weight in neonates of exercising mothers. ${ }^{38}$

\section{Association between Maternal Physical Activity and Newborn's Body Composition}

Several studies reported more than one birth weight outcome, but only two did report on the association between maternal physical activity and infant's body composition. ${ }^{36,75}$ Clapp and Capeless $^{36}$ compared a group of recreational athletes pursuing a heavy exercise regimen throughout pregnancy to a group of matched active women not involved in high-intensity training. Skinfold thickness, fat mass, and body fat percentage of the newborns were all significantly reduced in the heavy exercise group as compared with the comparison group, with a difference in fat mass of $220 \mathrm{~g}$ between groups. Similarly, Harrod et $\mathrm{al}^{75}$ observed a significant decrease in neonatal fat mass with increased total physical activity during 


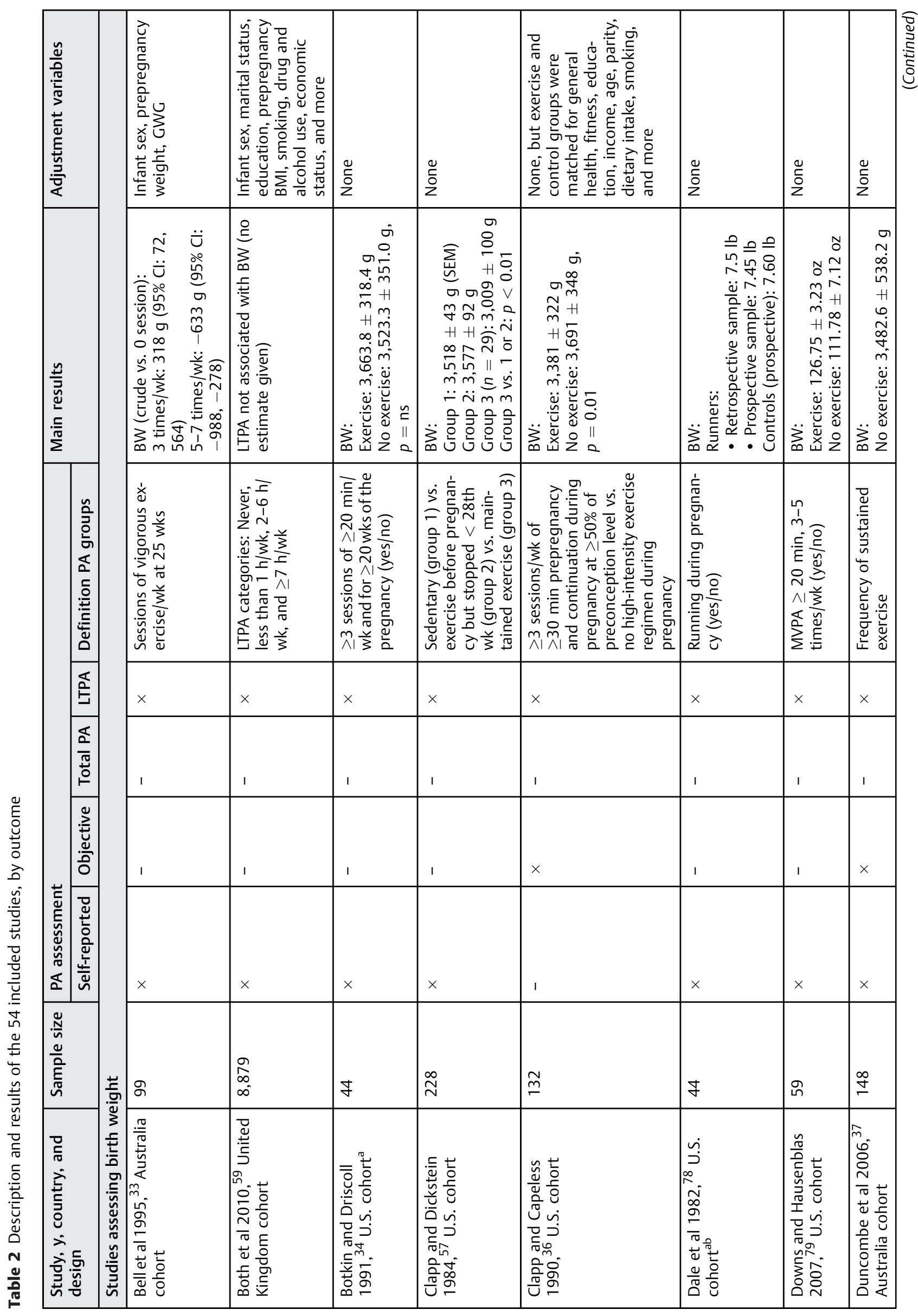




\begin{tabular}{|c|c|c|c|c|}
\hline 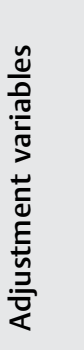 & & 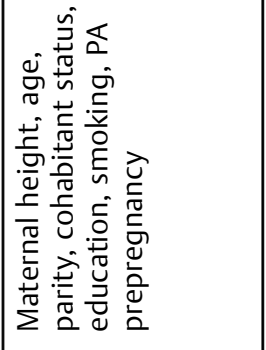 & 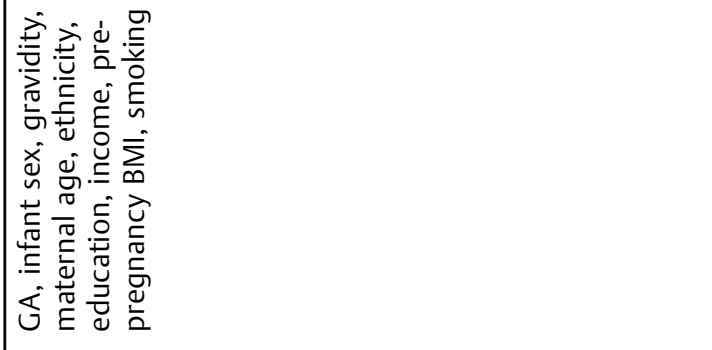 & 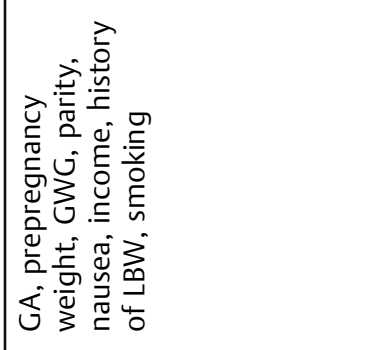 \\
\hline 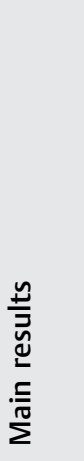 & 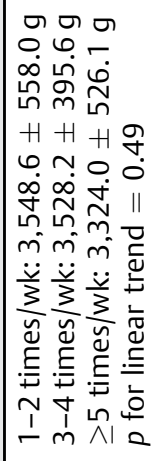 & 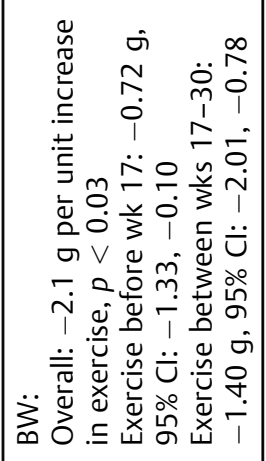 & 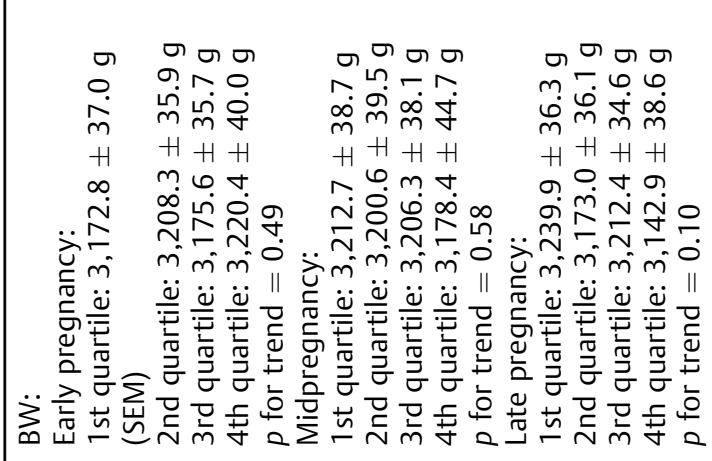 & 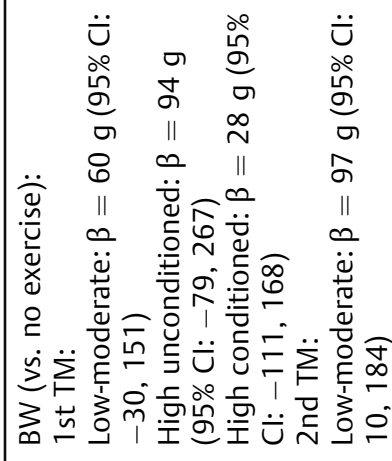 \\
\hline 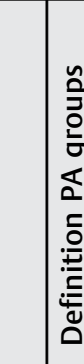 & & 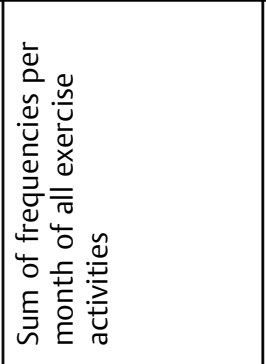 & 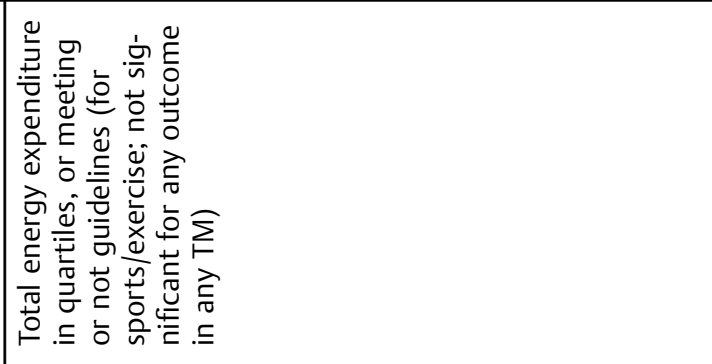 & 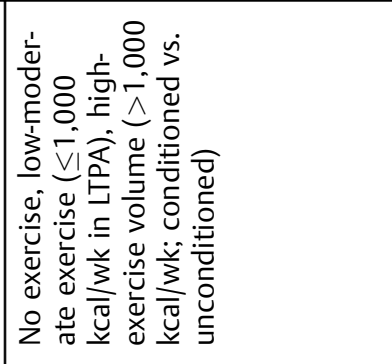 \\
\hline 皇 & & $\times$ & $x$ & $x$ \\
\hline $\mid \begin{array}{l}\frac{\nwarrow}{0} \\
\frac{\pi}{0} \\
\circ\end{array}$ & & 1 & $x$ & 1 \\
\hline | & & 1 & 1 & 1 \\
\hline 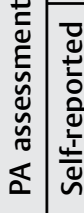 & & $x$ & $x$ & $x$ \\
\hline $\begin{array}{l}\frac{\tilde{N}}{n} \\
\frac{\tilde{U}}{0} \\
\frac{\vec{E}}{\tilde{N}}\end{array}$ & & $\begin{array}{l}\stackrel{n}{\circ} \\
\text { r } \\
\text { r }\end{array}$ & 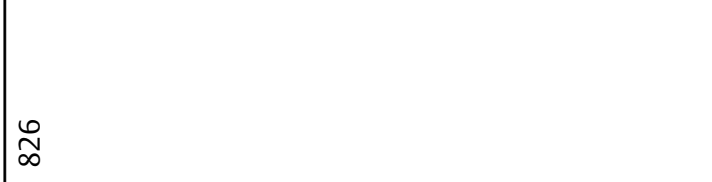 & Oo \\
\hline 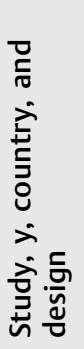 & & 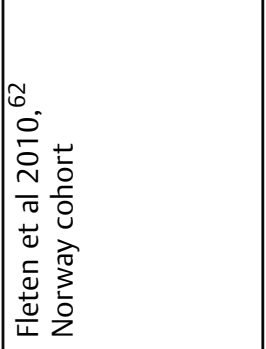 & 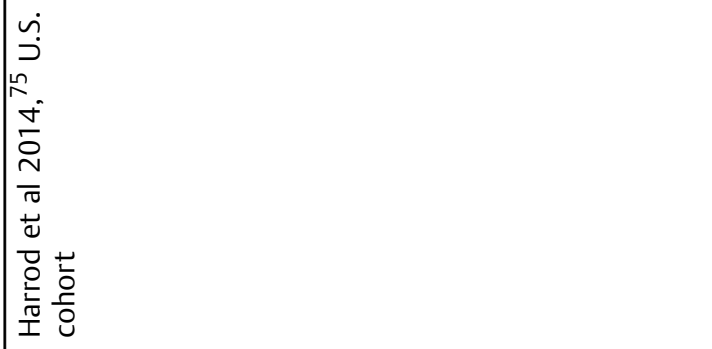 & 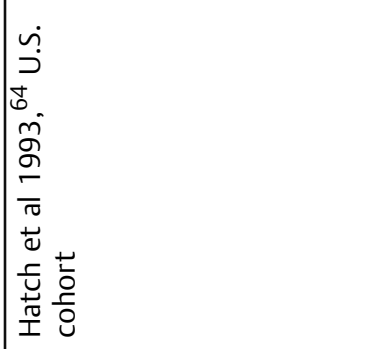 \\
\hline
\end{tabular}




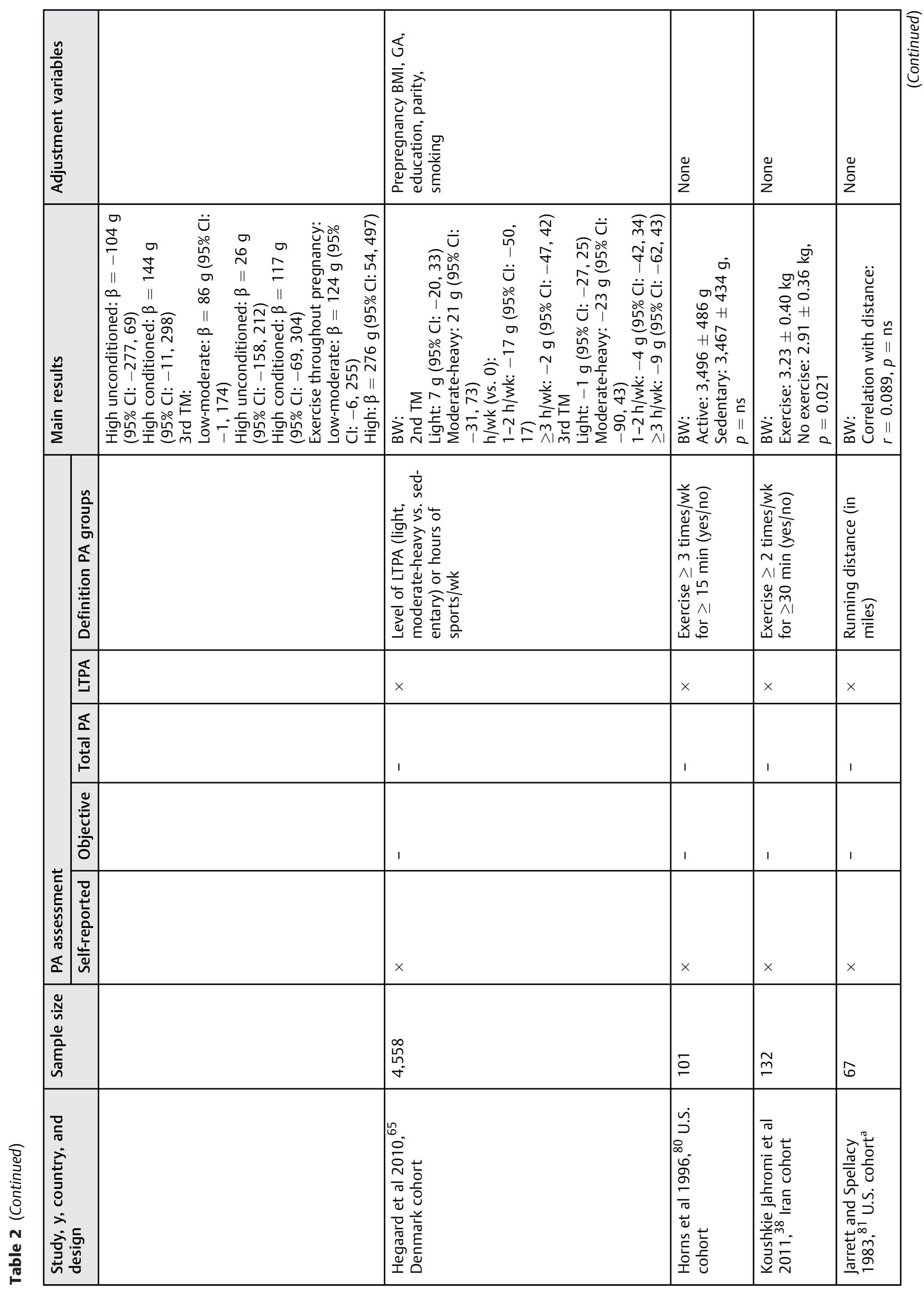




\begin{tabular}{|c|c|c|c|c|c|}
\hline 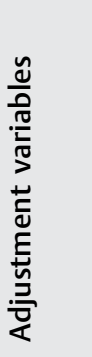 & 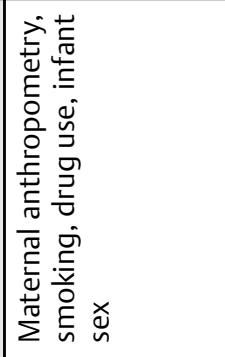 & 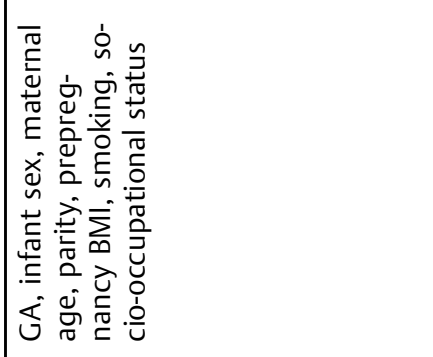 & 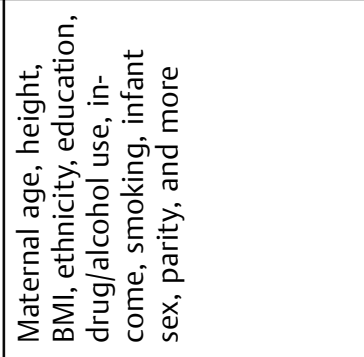 & 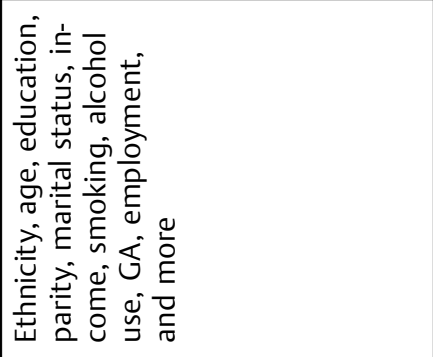 & 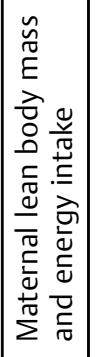 \\
\hline 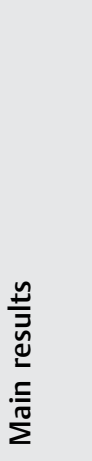 & 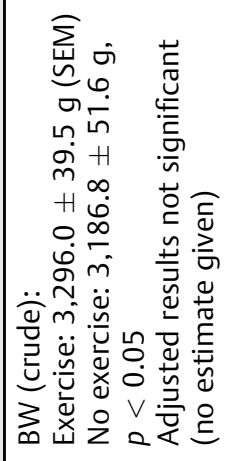 & 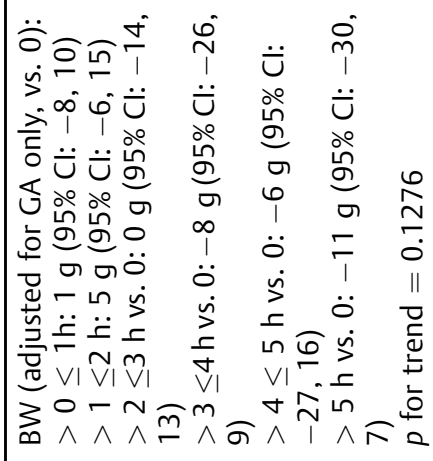 & 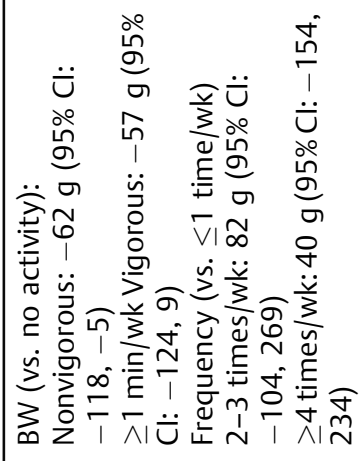 & 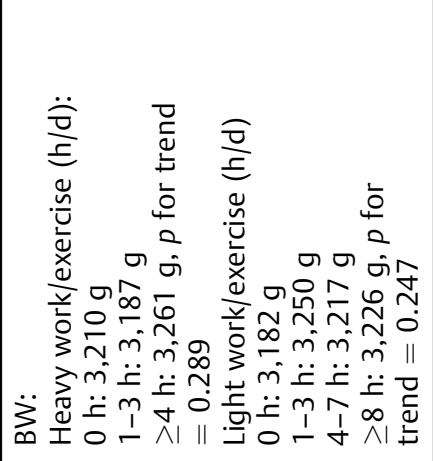 & 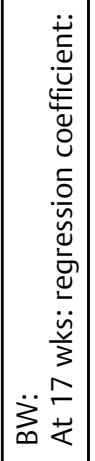 \\
\hline 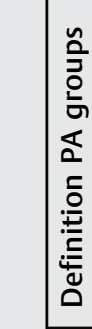 & 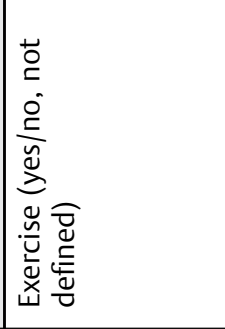 & 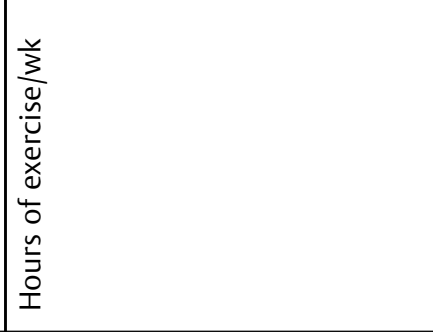 & 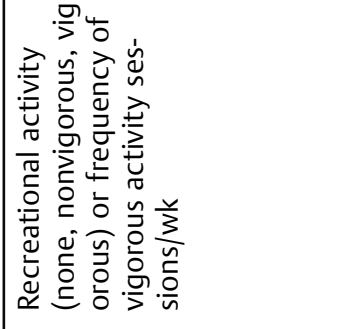 & 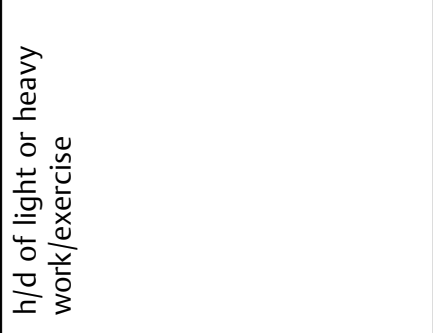 & 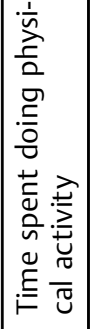 \\
\hline 㝴 & $\times$ & $\times$ & $\times$ & $x$ & 1 \\
\hline $\begin{array}{l}\frac{\pi}{2} \\
\frac{\pi}{0} \\
\stackrel{0}{\circ}\end{array}$ & 1 & 1 & $\times$ & $x$ & $\times$ \\
\hline 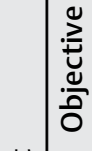 & 1 & 1 & 1 & 1 & $x$ \\
\hline 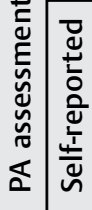 & $x$ & $x$ & $x$ & $x$ & \\
\hline 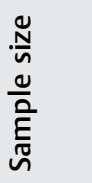 & $\underset{\sim}{\stackrel{\sim}{\sim}}$ & 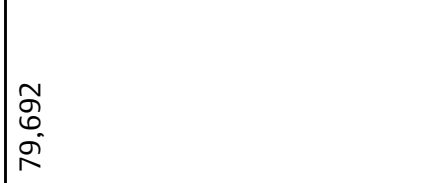 & $\stackrel{\infty}{\rightleftharpoons}$ & 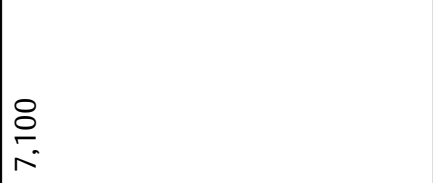 & in \\
\hline 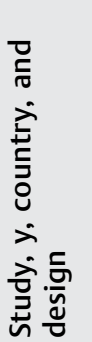 & 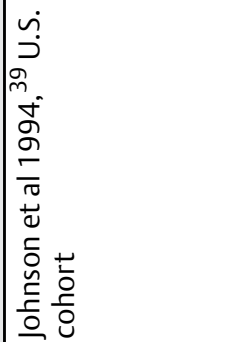 & 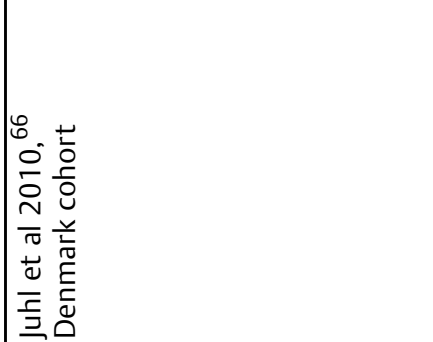 & 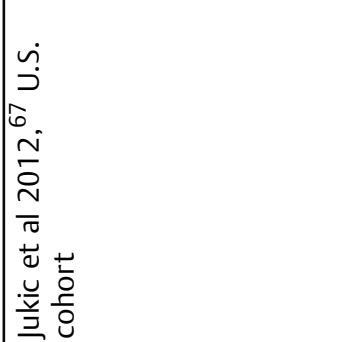 & 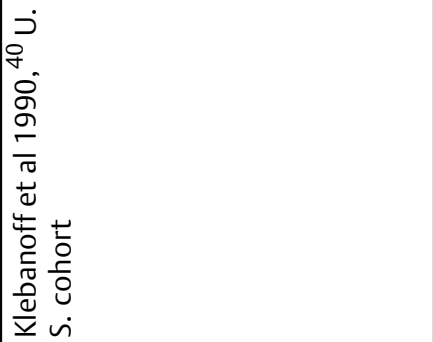 & 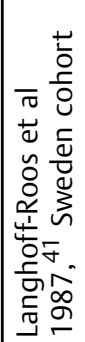 \\
\hline
\end{tabular}


Maternal Physical Activity and Birth Weight Bisson et al. e179

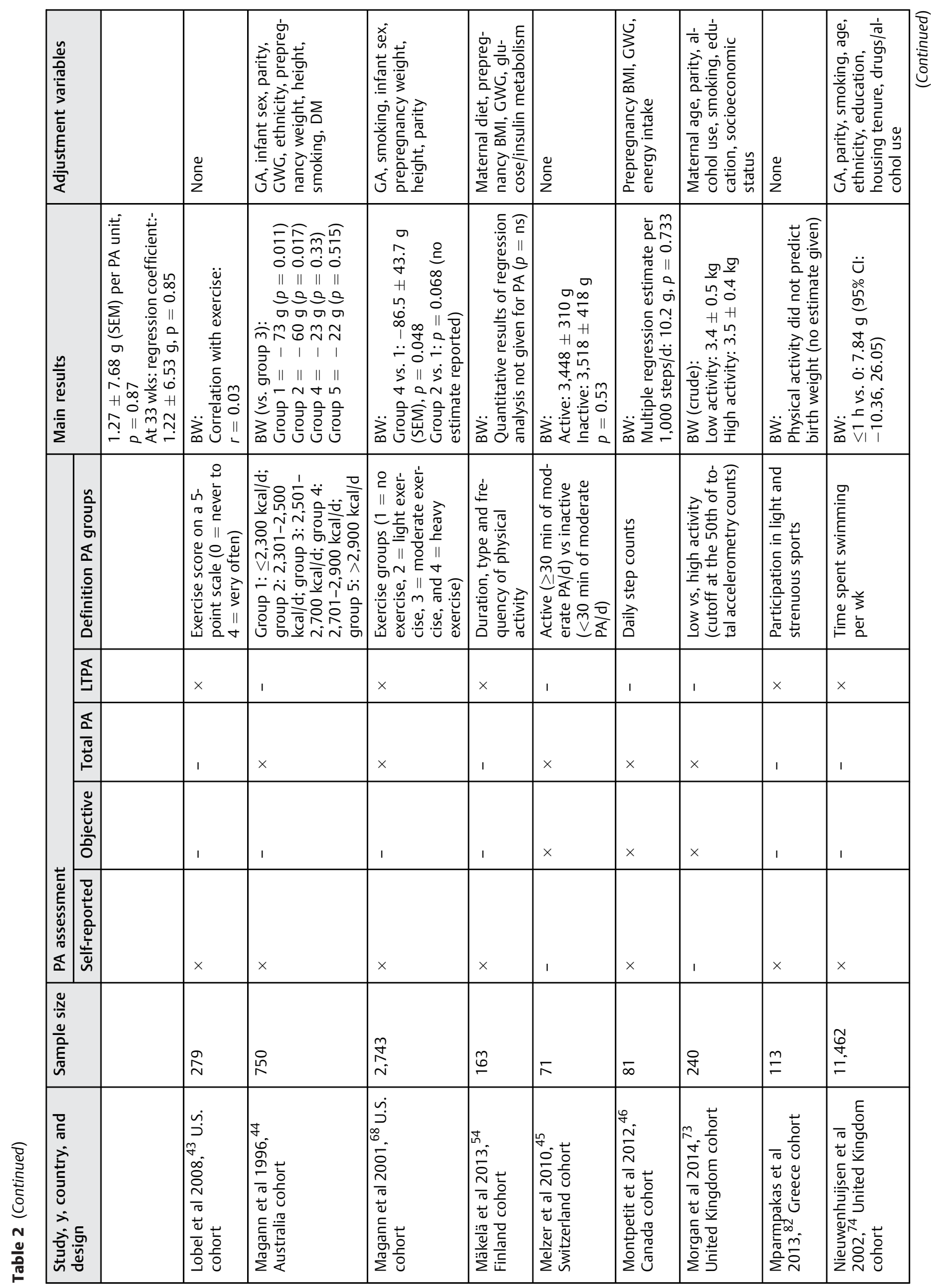




\begin{tabular}{|c|c|c|c|c|c|c|c|}
\hline 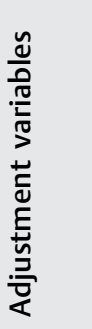 & & $\begin{array}{l}\mathscr{I} \\
\text { Oे }\end{array}$ & $\mathbb{\Xi}$ & $\mid \begin{array}{l}0 \\
\check{0} \\
z\end{array}$ & 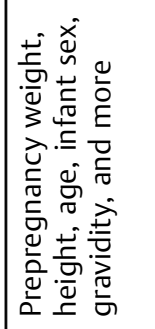 & 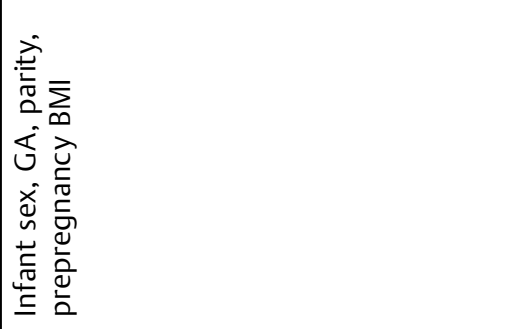 & 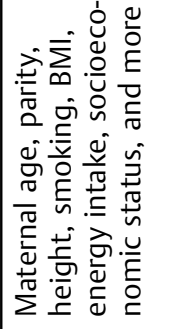 \\
\hline 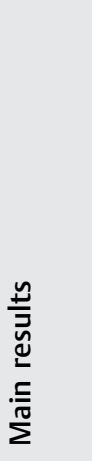 & 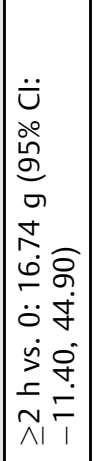 & 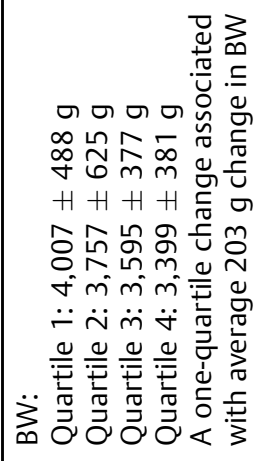 & 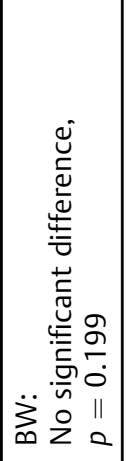 & 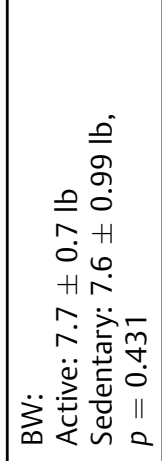 & 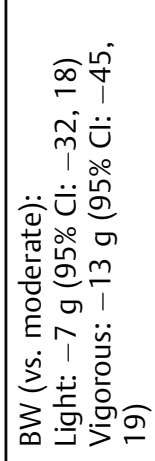 & 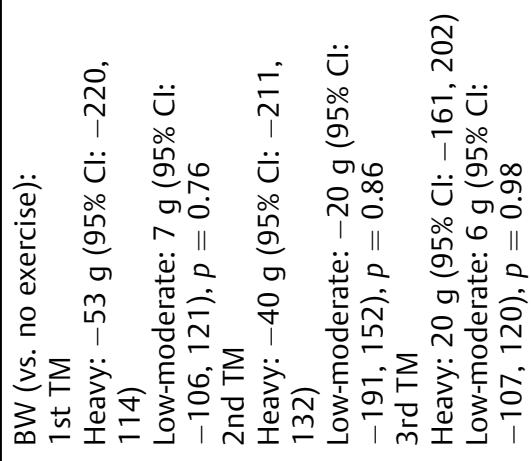 & 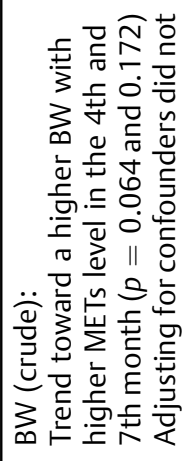 \\
\hline 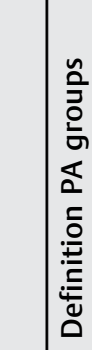 & & 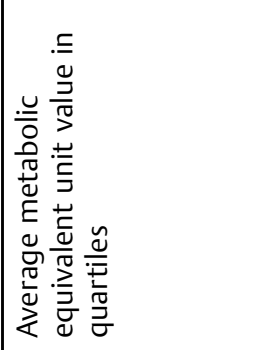 & 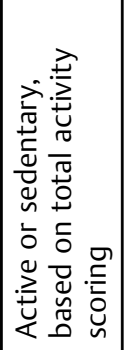 & 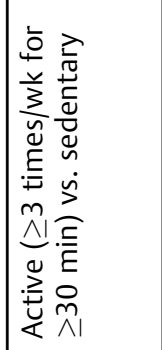 & 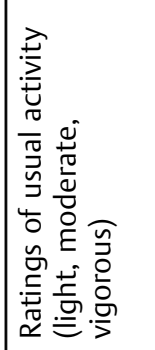 & 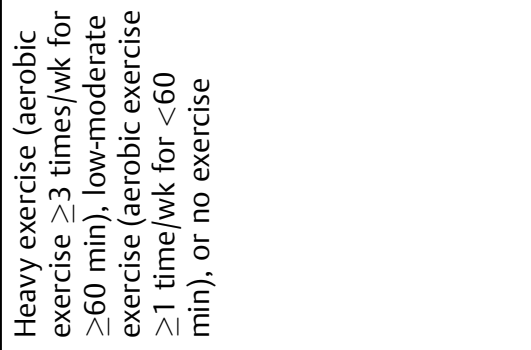 & 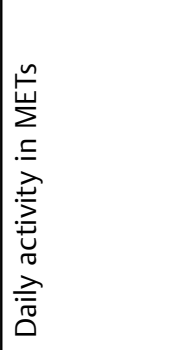 \\
\hline 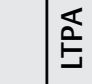 & & 1 & 1 & $x$ & $x$ & $x$ & 1 \\
\hline $\begin{array}{l}\frac{\pi}{2} \\
\frac{\pi}{0} \\
0\end{array}$ & & $\times$ & $x$ & 1 & 1 & 1 & $x$ \\
\hline+ & & $\times$ & 1 & 1 & 1 & 1 & 1 \\
\hline 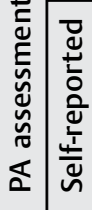 & & $\times$ & $x$ & $x$ & $\times$ & $x$ & $x$ \\
\hline $\begin{array}{l}\frac{\tilde{N}}{n} \\
\frac{0}{0} \\
\frac{E}{n} \\
\end{array}$ & & โn & เ & $\stackrel{\sim}{\sim}$ & $\begin{array}{l}\stackrel{\mathcal{N}}{m} \\
\stackrel{N}{N}\end{array}$ & $\mid \begin{array}{c}\infty \\
\infty \\
m\end{array}$ & 㔯 \\
\hline 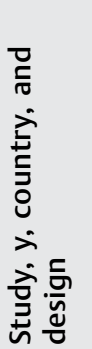 & & 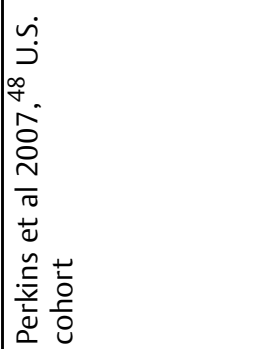 & 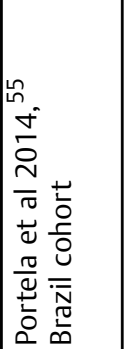 & 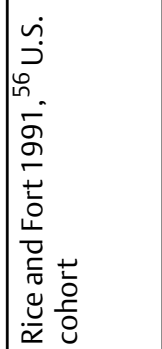 & 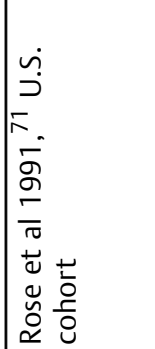 & 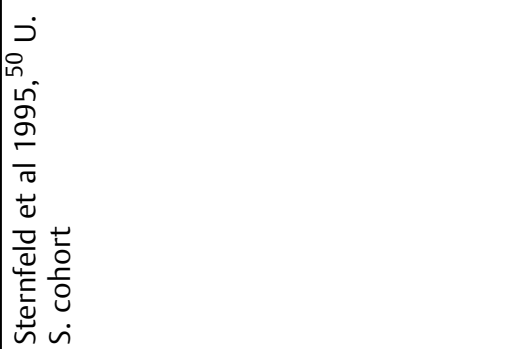 & 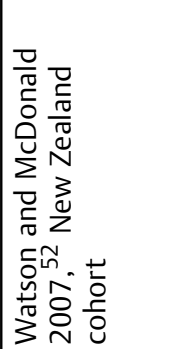 \\
\hline
\end{tabular}




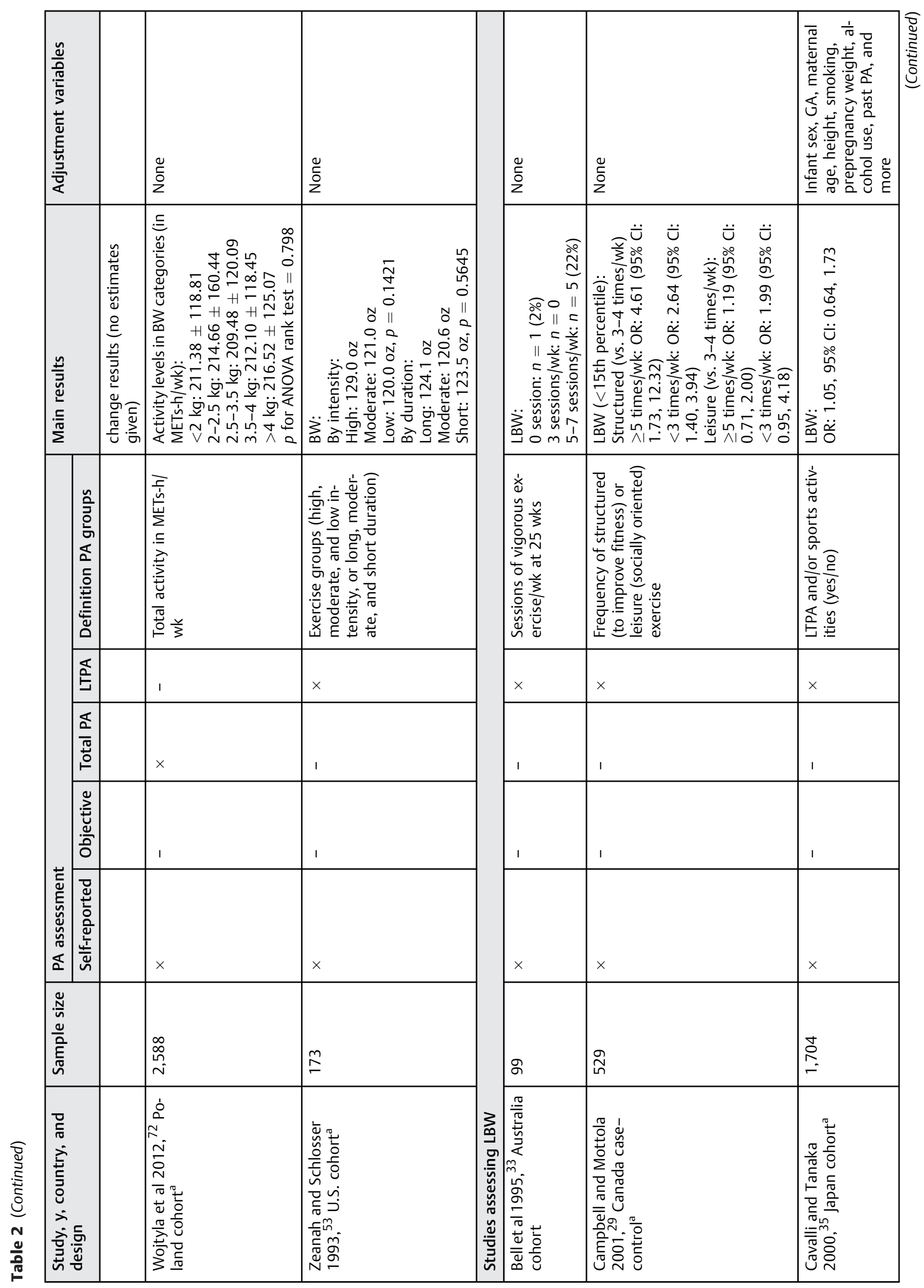




\begin{tabular}{|c|c|c|c|c|c|c|}
\hline 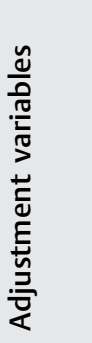 & 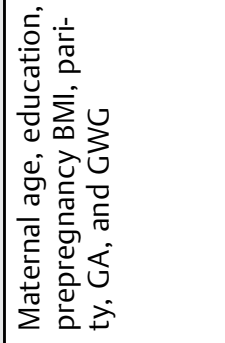 & 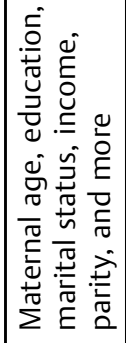 & $\mid \begin{array}{l}\tilde{y} \\
\text { ż }\end{array}$ & 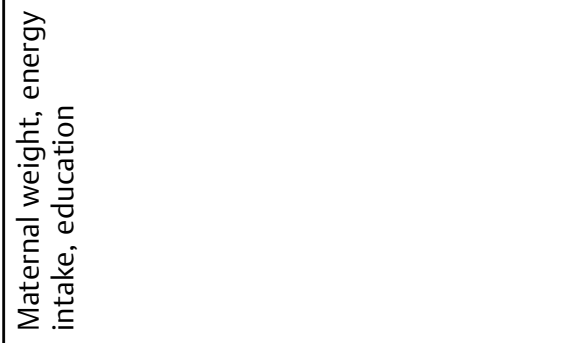 & 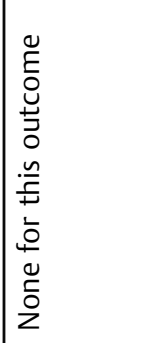 & 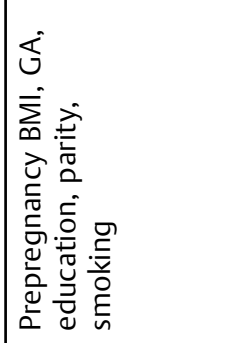 \\
\hline 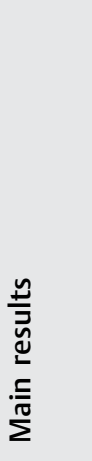 & 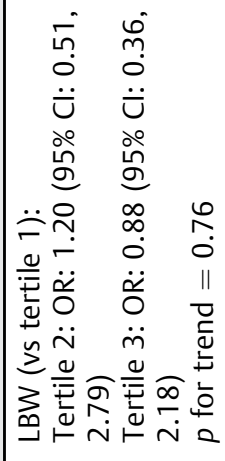 & 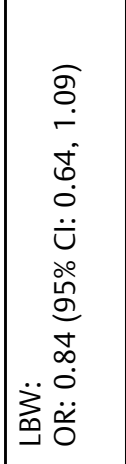 & 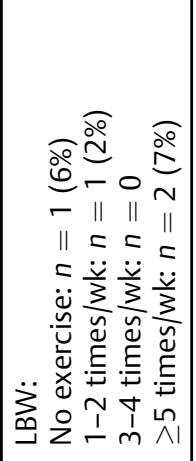 & 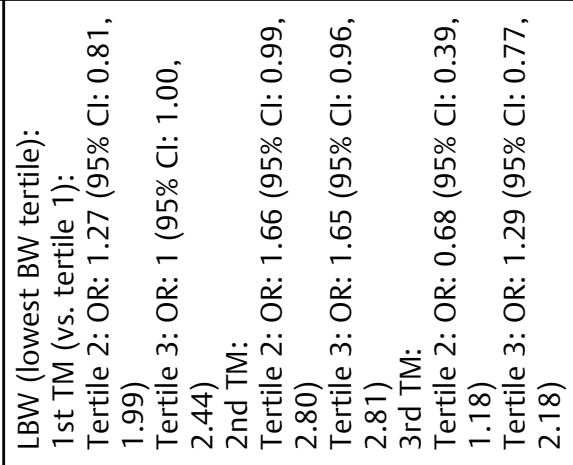 & 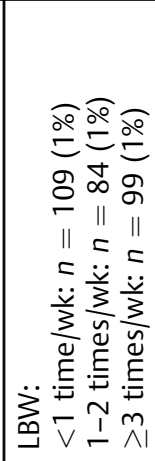 & 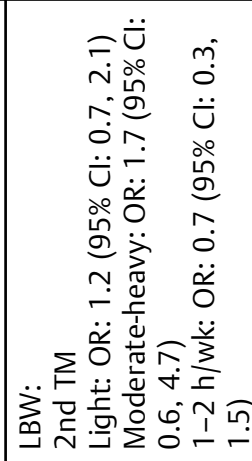 \\
\hline 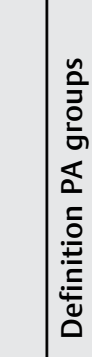 & 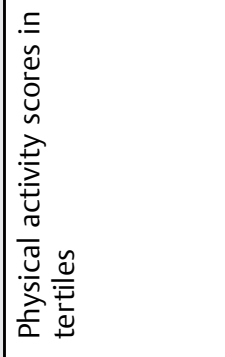 & 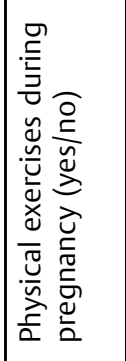 & 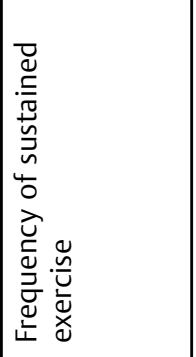 & 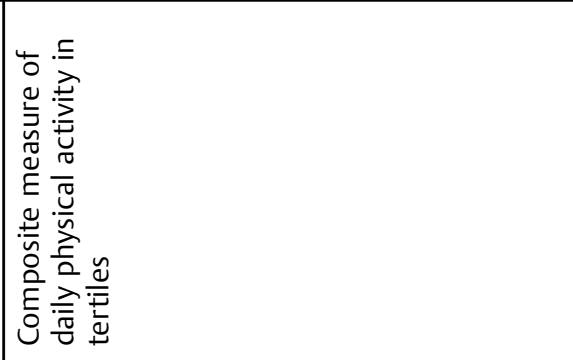 & 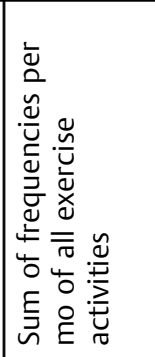 & 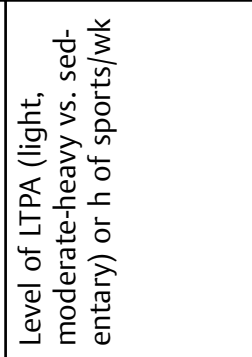 \\
\hline 选 & 1 & $x$ & $x$ & 1 & $\times$ & $x$ \\
\hline $\begin{array}{l}\frac{\pi}{2} \\
\frac{\pi}{0} \\
\stackrel{0}{\circ}\end{array}$ & $\times$ & 1 & 1 & $\times$ & 1 & 1 \\
\hline 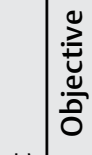 & 1 & 1 & $x$ & 1 & 1 & 1 \\
\hline 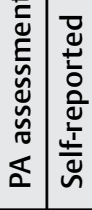 & $\times$ & $\times$ & $x$ & $\times$ & $\times$ & $\times$ \\
\hline 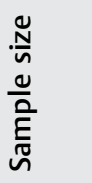 & $\frac{9}{i}$ & $\mid \begin{array}{l}\hat{n} \\
\hat{n} \\
\sim\end{array}$ & $\underset{+}{\stackrel{\infty}{+}}$ & $\begin{array}{l}0 \\
\text { L }\end{array}$ & 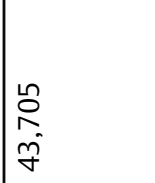 & $\begin{array}{l}\infty \\
\stackrel{\infty}{n} \\
\stackrel{n}{\gamma}\end{array}$ \\
\hline 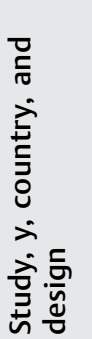 & 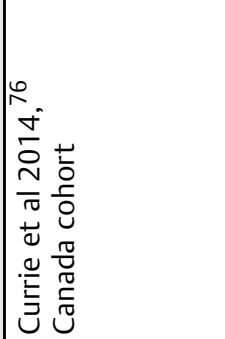 & 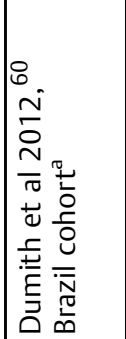 & 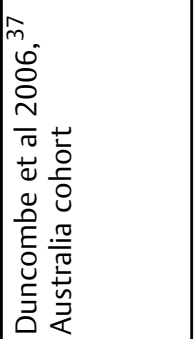 & 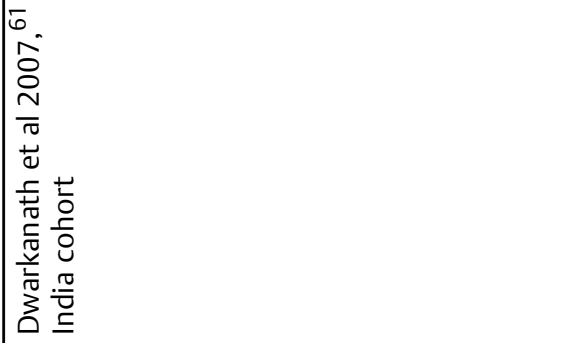 & 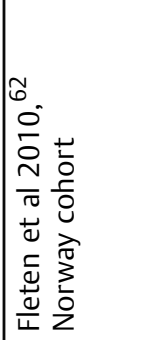 & 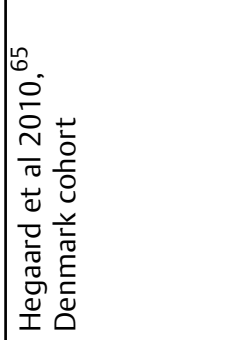 \\
\hline
\end{tabular}


Maternal Physical Activity and Birth Weight Bisson et al. e183

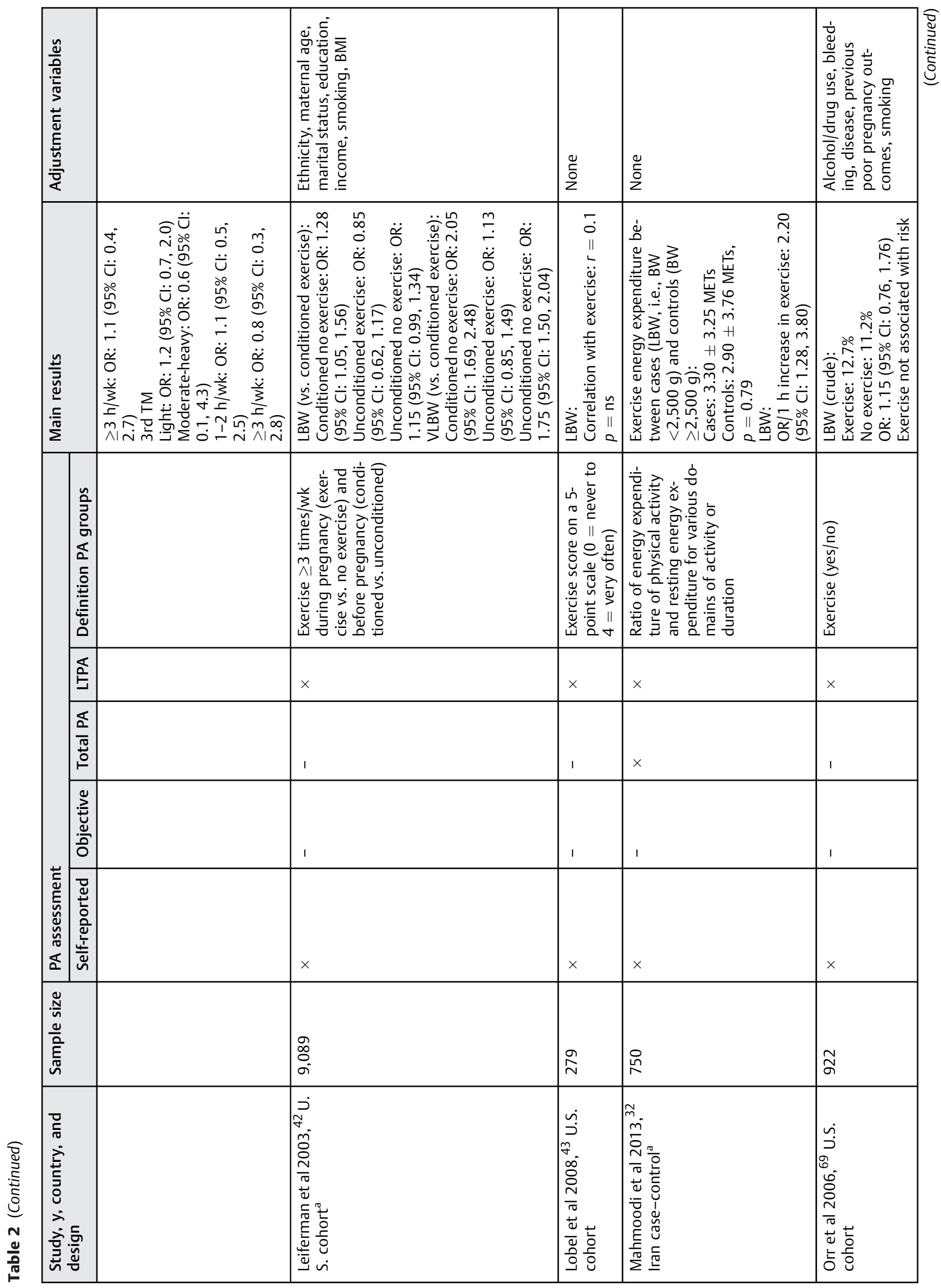




\begin{tabular}{|c|c|c|c|c|c|c|c|}
\hline 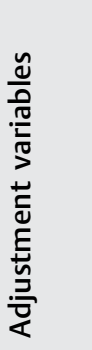 & & 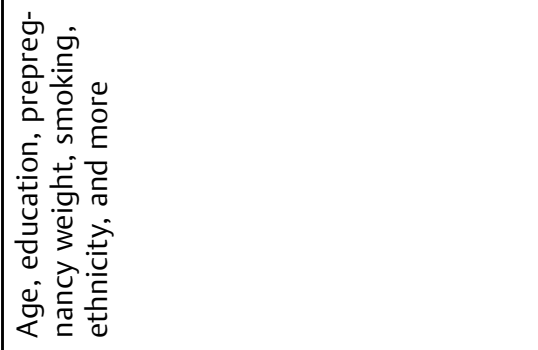 & 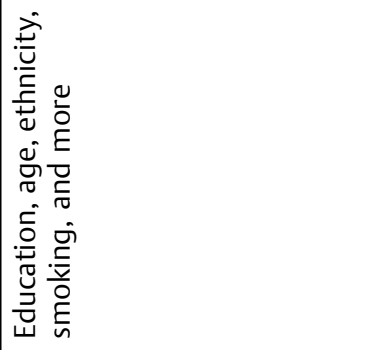 & & 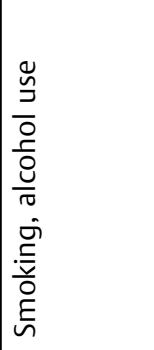 & $\mid \begin{array}{l}\mathscr{I} \\
\text { Oे }\end{array}$ & 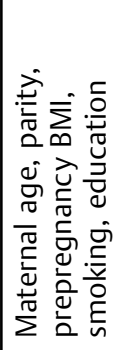 \\
\hline 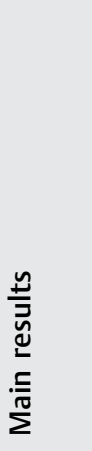 & 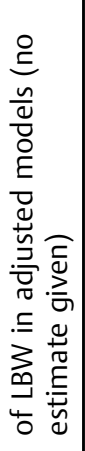 & 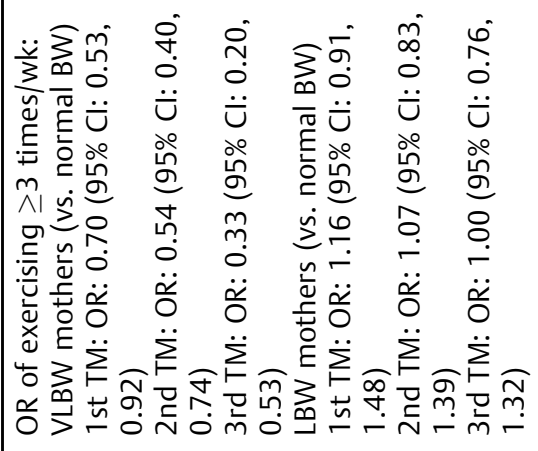 & 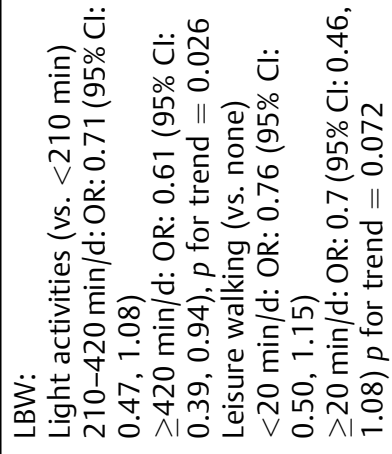 & & 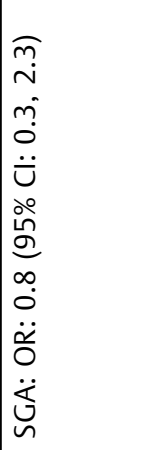 & 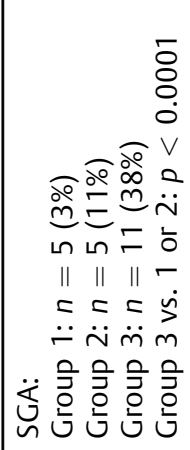 & 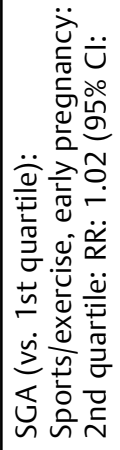 \\
\hline 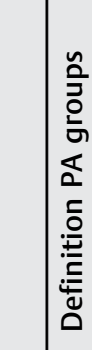 & & 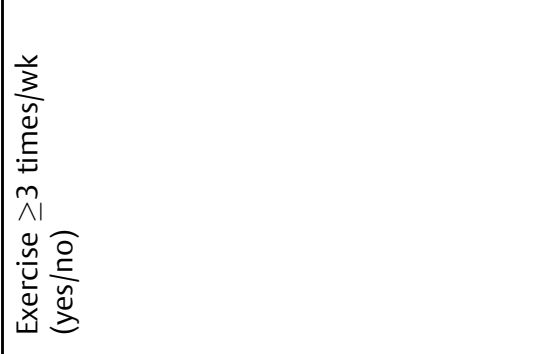 & 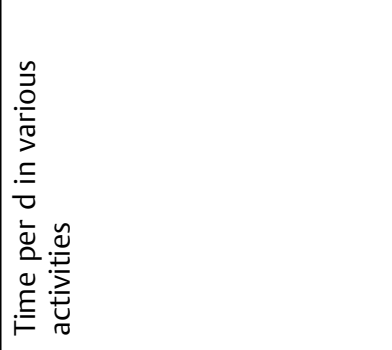 & & 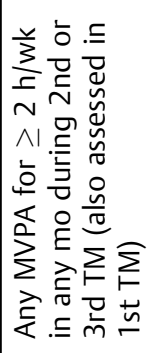 & 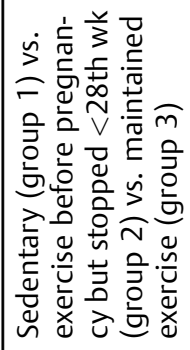 & 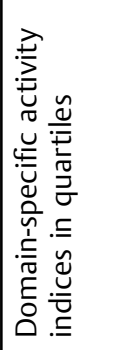 \\
\hline 㝴 & & $x$ & $x$ & & 1 & $x$ & $x$ \\
\hline 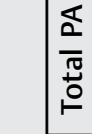 & & 1 & 1 & & $x$ & 1 & $x$ \\
\hline 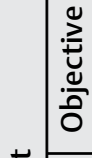 & & 1 & 1 & & 1 & 1 & 1 \\
\hline 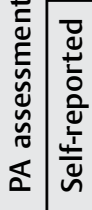 & & $\times$ & $x$ & & $x$ & $\times$ & $x$ \\
\hline $\begin{array}{l}\frac{\tilde{N}}{\tilde{n}} \\
\frac{0}{\hat{a}} \\
\frac{E}{\tilde{N}}\end{array}$ & & $\mid \begin{array}{l}\infty \\
\tilde{m} \\
\sim \\
\sim\end{array}$ & $\frac{\sigma}{\infty}$ & & হ্ন & $\stackrel{\sim}{N}$ & 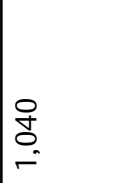 \\
\hline 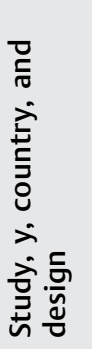 & & 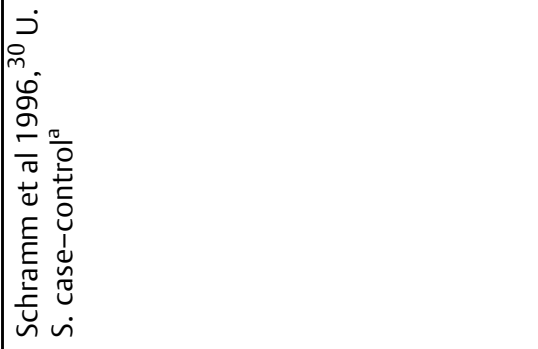 & 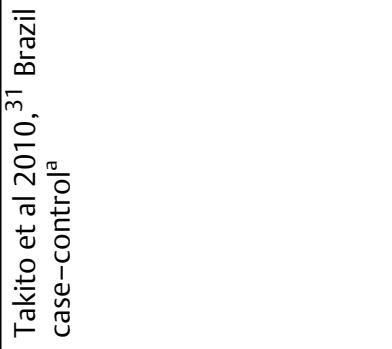 & 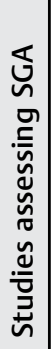 & 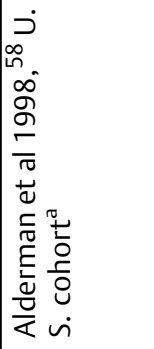 & 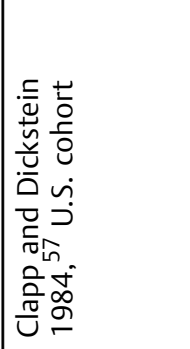 & 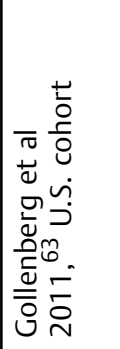 \\
\hline
\end{tabular}




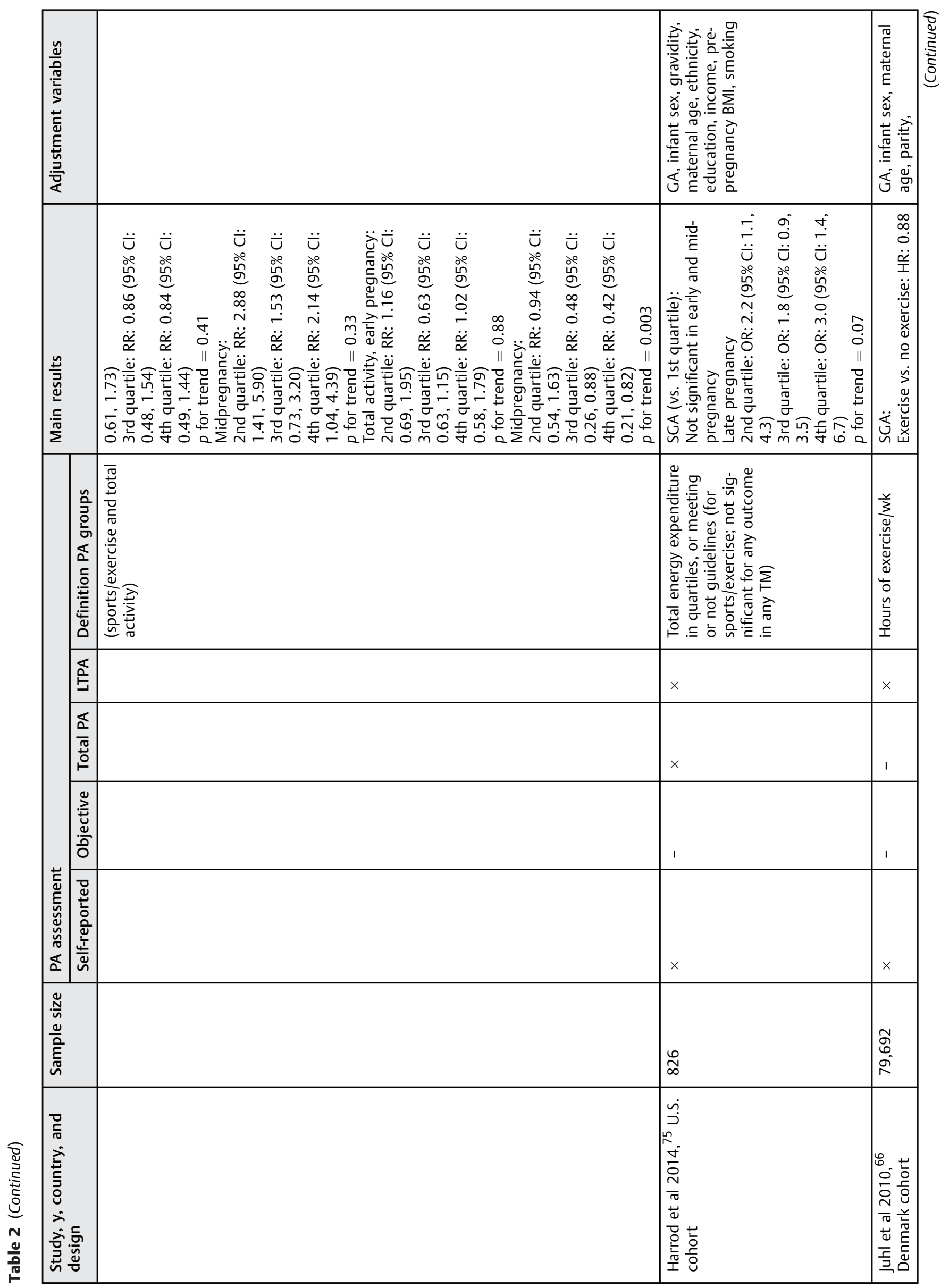




\begin{tabular}{|c|c|c|c|c|c|}
\hline 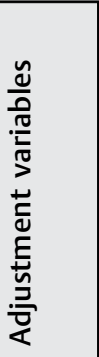 & 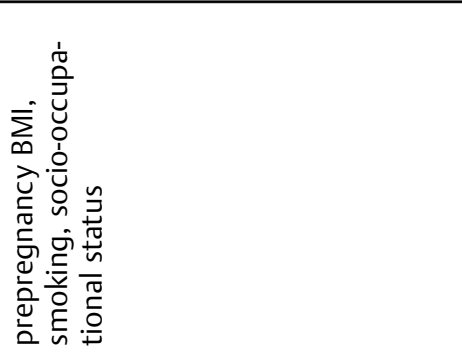 & 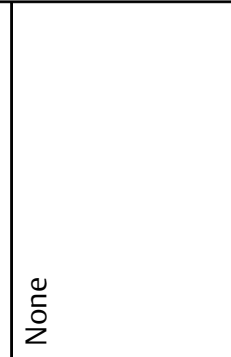 & 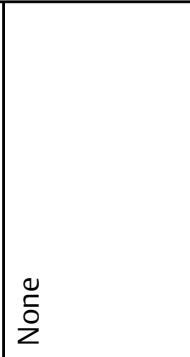 & 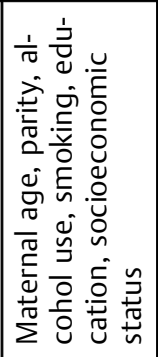 & 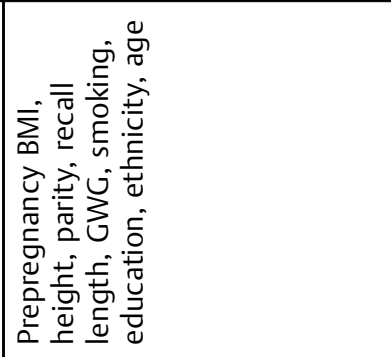 \\
\hline 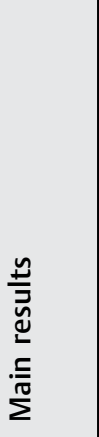 & 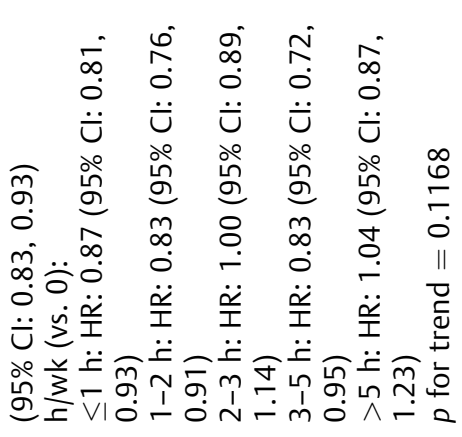 & 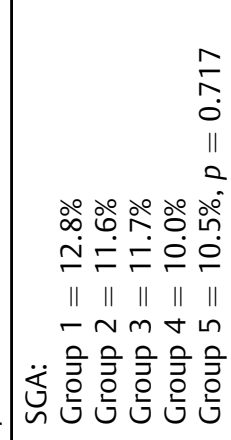 & 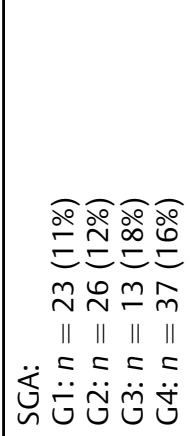 & 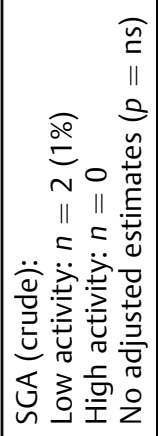 & 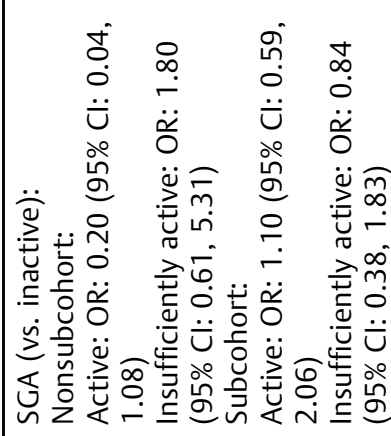 \\
\hline 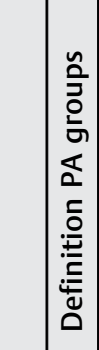 & & 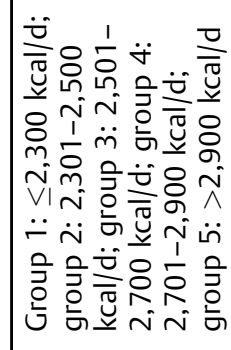 & 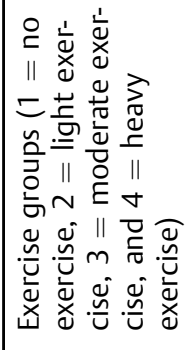 & 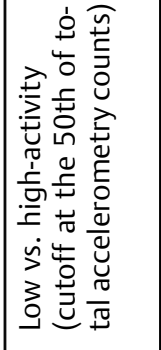 & 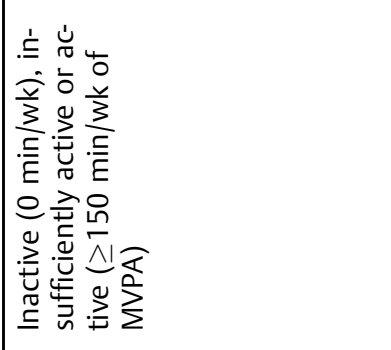 \\
\hline 起 & & 1 & $x$ & 1 & $\times$ \\
\hline 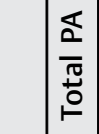 & & $x$ & $\times$ & $\times$ & 1 \\
\hline 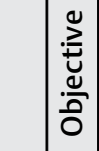 & & 1 & 1 & $\times$ & I \\
\hline 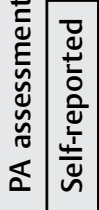 & & $x$ & $x$ & 1 & $\times$ \\
\hline 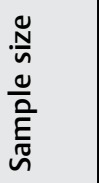 & & 욧 & $\frac{m}{\stackrel{m}{\sim}}$ & $\stackrel{\stackrel{P}{\sim}}{\sim}$ & $\underbrace{+}_{-}$ \\
\hline 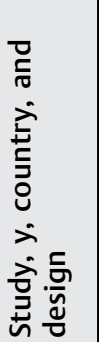 & & 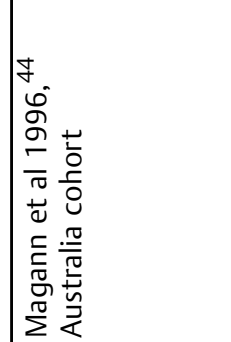 & 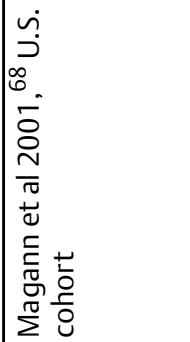 & 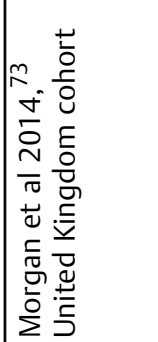 & 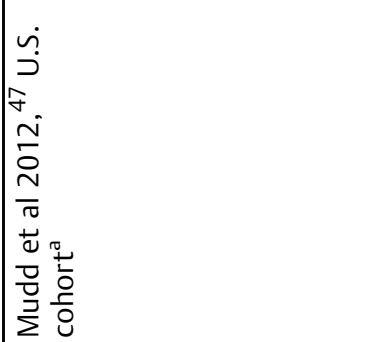 \\
\hline
\end{tabular}




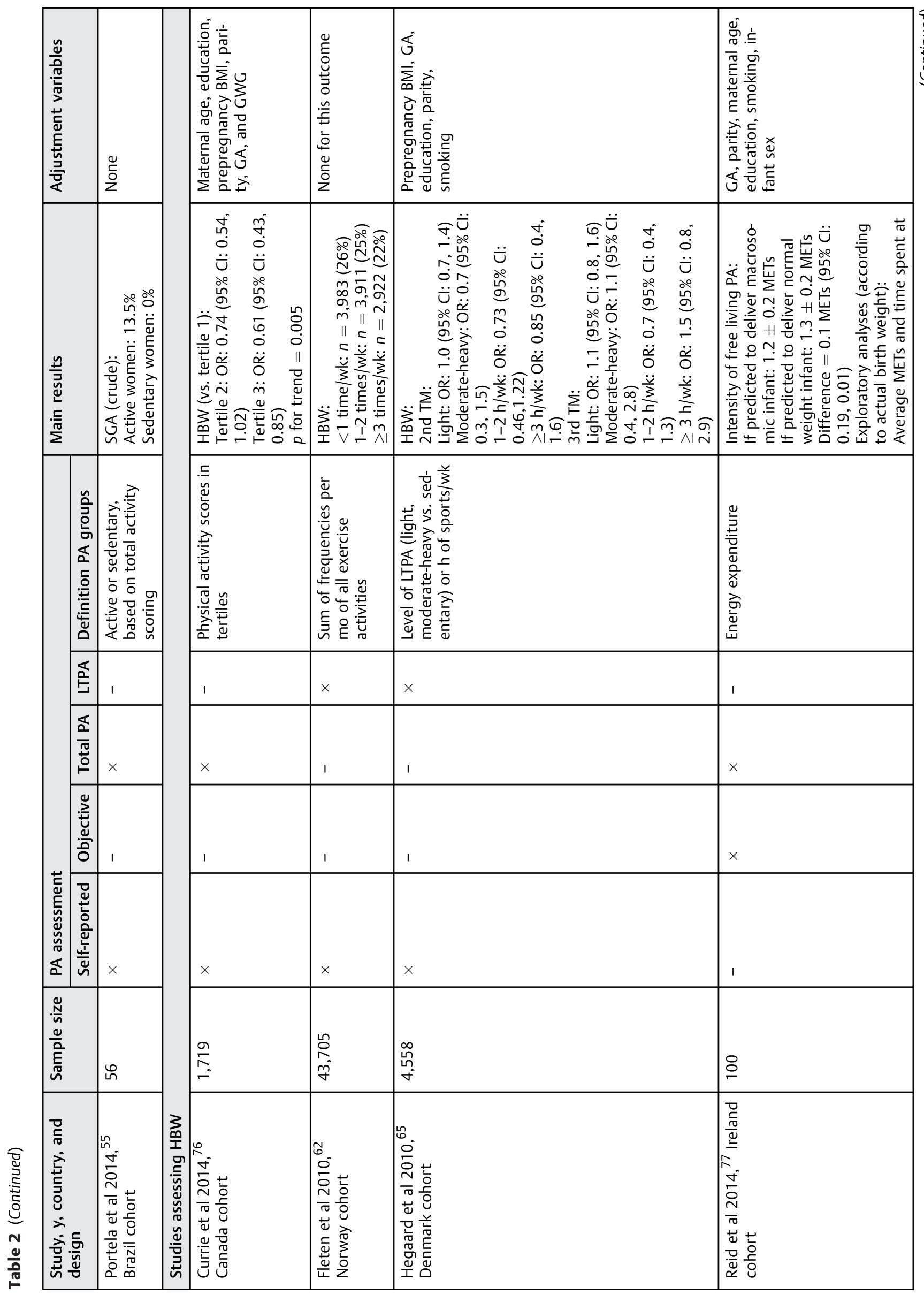




\begin{tabular}{|c|c|c|c|c|c|c|}
\hline 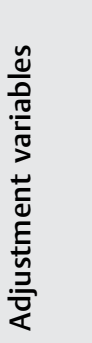 & & $\begin{array}{l}\text { İ } \\
\text { о }\end{array}$ & 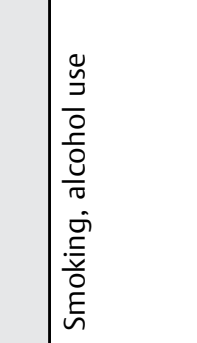 & $\mid \begin{array}{l}0 \\
\text { o } \\
z\end{array}$ & 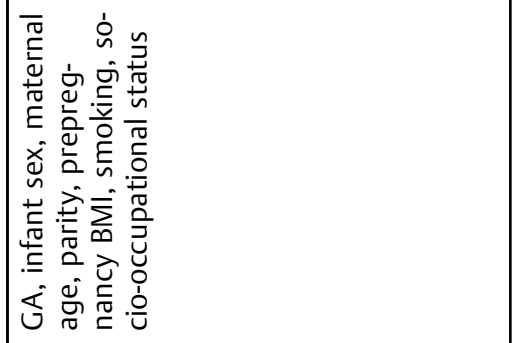 & 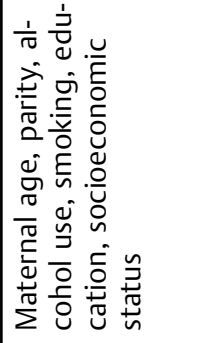 \\
\hline 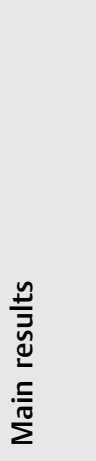 & 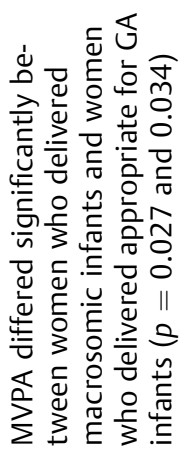 & 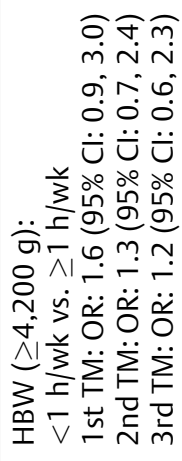 & 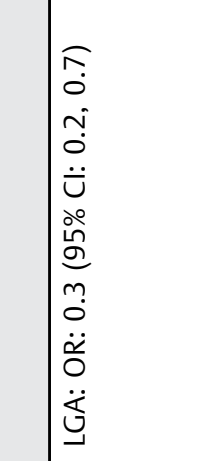 & 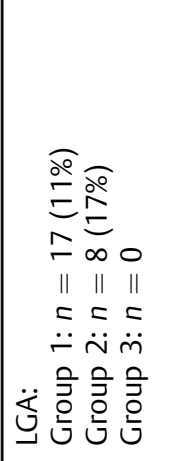 & 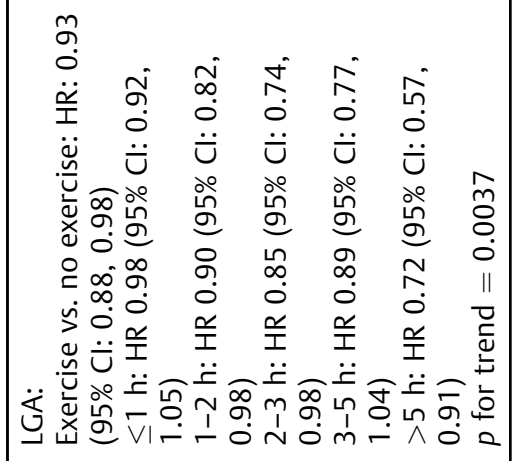 & 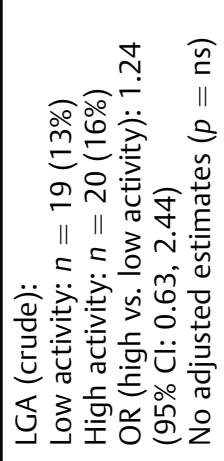 \\
\hline 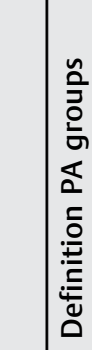 & & 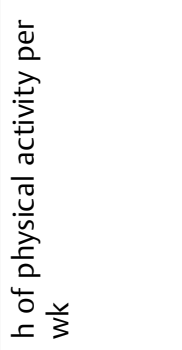 & 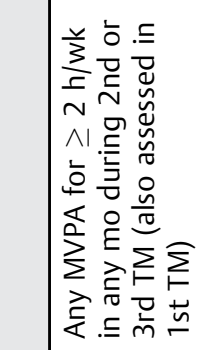 & 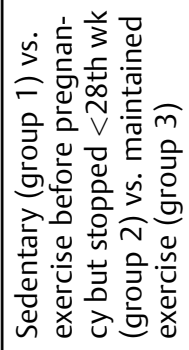 & 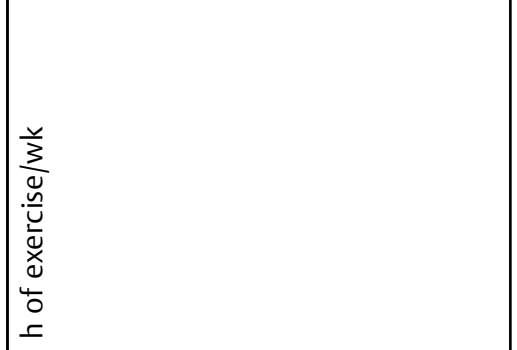 & 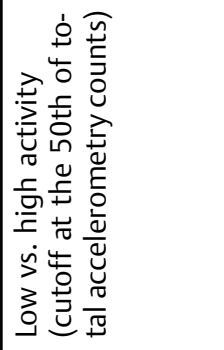 \\
\hline$\underline{\underline{\Xi}}$ & & $\times$ & 1 & $x$ & $x$ & 1 \\
\hline$\frac{\nwarrow}{\frac{\pi}{0}}$ & & 1 & $\times$ & 1 & 1 & $\times$ \\
\hline 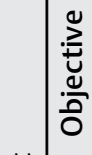 & & 1 & 1 & 1 & 1 & $x$ \\
\hline 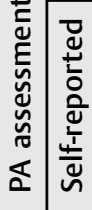 & & $x$ & $x$ & $x$ & $x$ & 1 \\
\hline 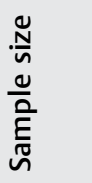 & & ڤ̂n & হ్ & $\stackrel{\infty}{\sim}$ & 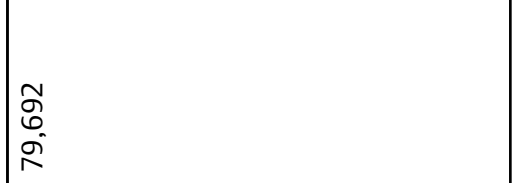 & $\stackrel{\stackrel{P}{\sim}}{\sim}$ \\
\hline 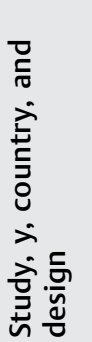 & & 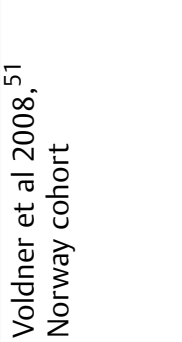 & 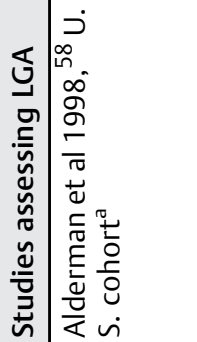 & 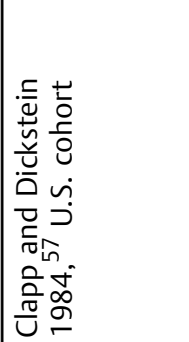 & 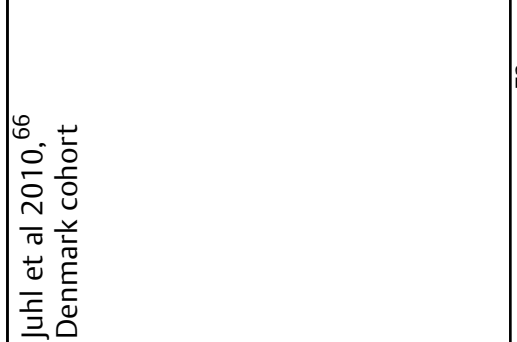 & 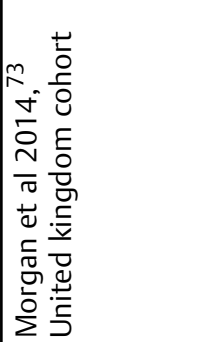 \\
\hline
\end{tabular}




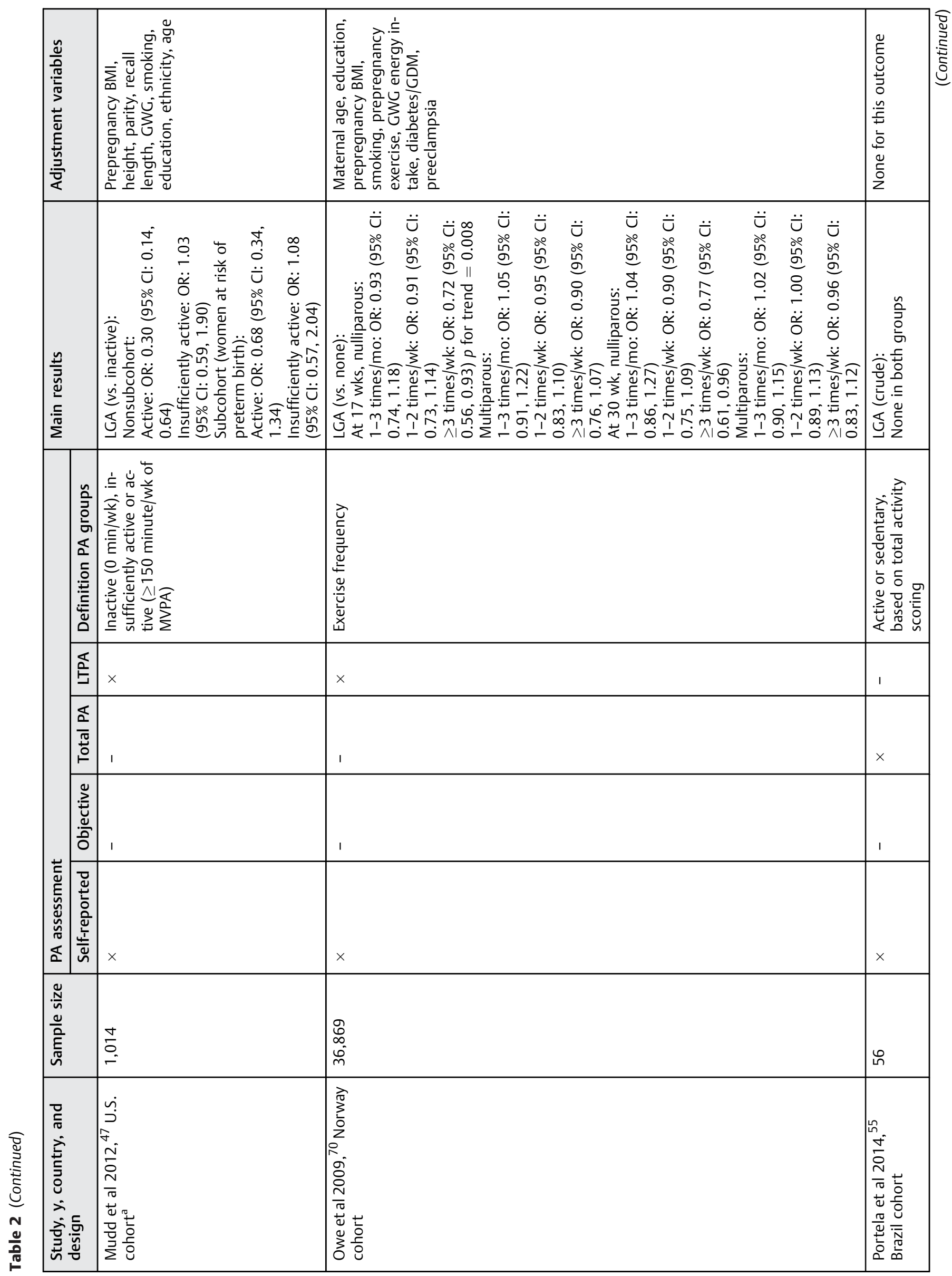




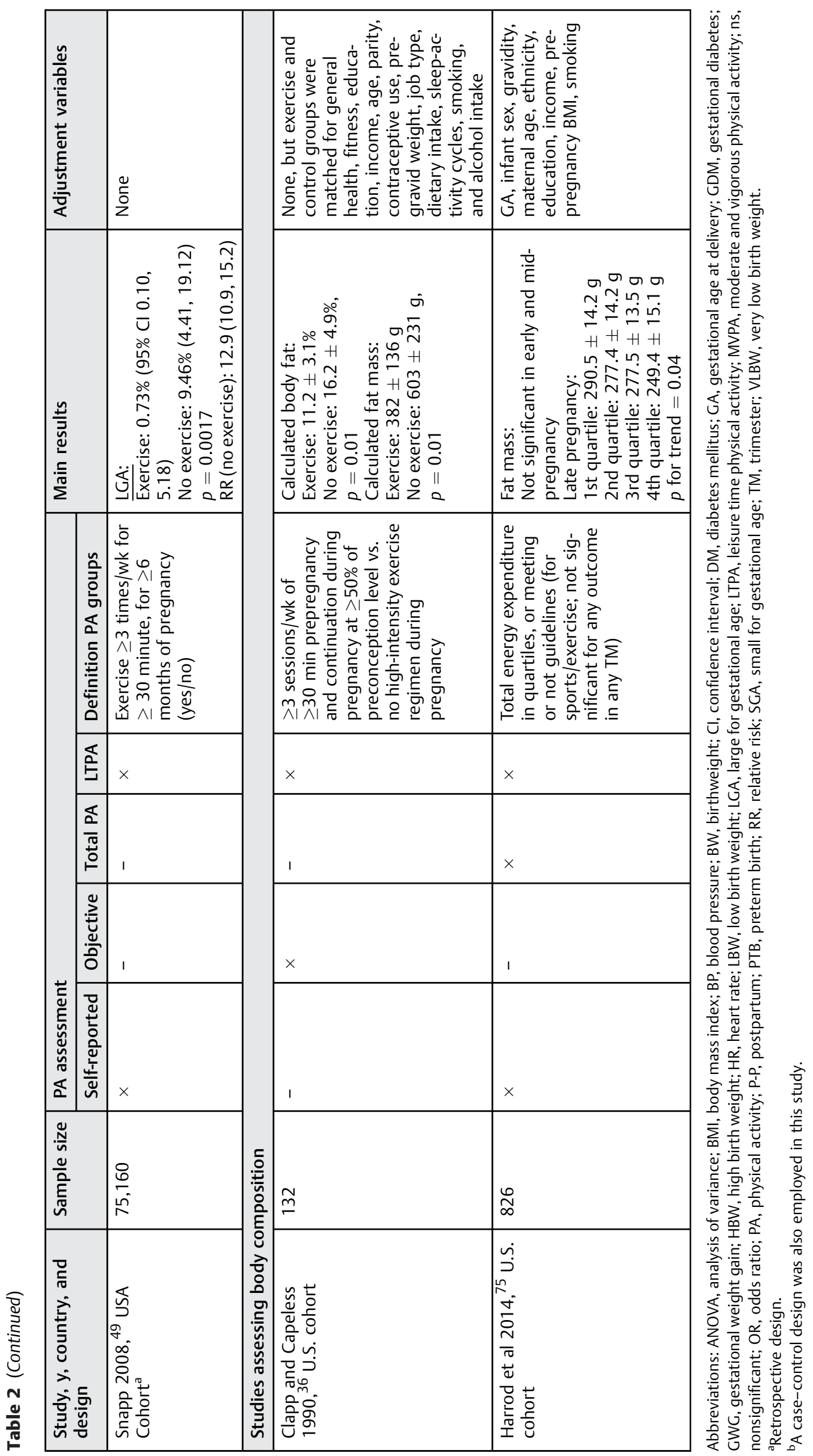




\begin{tabular}{|c|c|c|c|c|c|c|c|c|}
\hline Study or Subgroup & $\begin{array}{c}\text { Mean } \\
\text { Difference [g] }\end{array}$ & SE & $\begin{array}{l}\text { High level } \\
\text { Total }\end{array}$ & $\begin{array}{c}\text { Low leve } \\
\text { Total }\end{array}$ & Weight & $\begin{array}{l}\text { Mean Difference } \\
\text { Random, } 95 \% \mathrm{Cl}\end{array}$ & \multicolumn{2}{|c|}{ Random, $95 \% \mathrm{Cl}$} \\
\hline \multicolumn{7}{|c|}{2.1 High volume vs low volume - crude } & & \\
\hline Bell 1995 & -315 & 141 & 58 & 41 & $2.2 \%$ & $-315.00[-591.35,-38.65]$ & & \\
\hline Clapp 1984 & -509 & 108.8 & 29 & 152 & $3.3 \%$ & $-509.00[-722.35,-295.65]$ & & \\
\hline Clapp and Capeless 1990 & -310 & 59.5 & 77 & 55 & $7.0 \%$ & $-310.00[-426.62,-193.38]$ & $\longrightarrow$ & \\
\hline Duncombe 2006 & -158.6 & 165.1 & 27 & 17 & $1.7 \%$ & $-158.60[-482.38,165.18]$ & & \\
\hline Perkins 2007 & -608 & 174.4 & 12 & 13 & $1.5 \%$ & $-608.00[-949.82,-266.18]$ & & \\
\hline $\begin{array}{l}\text { Rice } 1991 \\
\text { Subtotal (95\% Cl) }\end{array}$ & 45.4 & 163.5 & $\begin{array}{r}12 \\
215\end{array}$ & $\begin{array}{r}11 \\
289\end{array}$ & $\begin{array}{r}1.7 \% \\
17.4 \%\end{array}$ & $\begin{array}{r}45.40[-275.05,365.85] \\
\mathbf{- 3 1 9 . 3 3}[-\mathbf{- 4 7 2 . 2 1 ,} \mathbf{- 1 6 6 . 4 5}]\end{array}$ & & \\
\hline \multicolumn{9}{|c|}{$\begin{array}{l}\text { Heterogeneity: } \text { Tau }^{2}=19373.36 ; \mathrm{Chi}^{2}=11.71, \mathrm{df}=5(\mathrm{P}=0.04) ; \mathrm{I}^{2}=57 \% \\
\text { Test for overall effect: } Z=4.09(P<0.0001)\end{array}$} \\
\hline \multicolumn{9}{|c|}{2.2 High volume vs low volume - adjusted } \\
\hline Harrod $2014^{a}$ & -97 & 52.9 & 206 & 206 & $7.7 \%$ & $-97.00[-200.68,6.68]$ & & \\
\hline Hatch 1993 & 276 & 113 & 15 & 185 & $3.1 \%$ & $276.00[54.52,497.48]$ & & \\
\hline $\begin{array}{l}\text { Magann } 2001 \\
\text { Subtotal }(95 \% \mathrm{Cl})\end{array}$ & -86.5 & 43.7 & $\begin{array}{l}238 \\
459\end{array}$ & $\begin{array}{l}217 \\
608\end{array}$ & $\begin{array}{r}8.9 \% \\
19.7 \%\end{array}$ & $\begin{array}{r}-86.50[-172.15,-0.85] \\
-5.00[-161.86,151.86]\end{array}$ & & \\
\hline \multicolumn{9}{|c|}{$\begin{array}{l}\text { Heterogeneity: } \text { Tau }^{2}=14416.18 ; \mathrm{Chi}^{2}=9.70, \mathrm{df}=2(P=0.008) ; \mathrm{P}^{2}=79 \% \\
\text { Test for overall effect: } Z=0.06(P=0.95)\end{array}$} \\
\hline \multicolumn{9}{|c|}{2.3 High duration vs low duration } \\
\hline Hegaard 2010 & -9 & 27.0 & 289 & 3672 & $11.1 \%$ & $-9.00[-62.00,44.00]$ & & $f$ \\
\hline Juhl 2010 & -11 & 9.4 & 2236 & 49929 & $12.8 \%$ & $-11.00[-29.42,7.42]$ & & 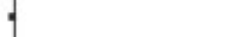 \\
\hline Nieuwenjuijsen $2002^{b}$ & 16.7 & 14.4 & 1131 & 6744 & $12.5 \%$ & $16.70[-11.52,44.92]$ & & $=$ \\
\hline $\begin{array}{l}\text { Sternfeld } 1995 \\
\text { Subtotal }(95 \% \mathrm{Cl})\end{array}$ & 20 & 92.6 & $\begin{array}{r}25 \\
3681\end{array}$ & $\begin{array}{r}122 \\
60467\end{array}$ & $\begin{array}{r}4.2 \% \\
40.5 \%\end{array}$ & $\begin{array}{r}20.00[-161.49,201.49] \\
-3.06[-17.82,11.70]\end{array}$ & & \\
\hline \multicolumn{9}{|c|}{$\begin{array}{l}\text { Heterogeneity: } \text { Tau }^{2}=0.00 ; \mathrm{Ch}^{2}=2.71, \mathrm{df}=3(\mathrm{P}=0.44) ; \mathrm{I}^{2}=0 \% \\
\text { Test for overall effect: } \mathrm{Z}=0.41(\mathrm{P}=0.68)\end{array}$} \\
\hline \multicolumn{9}{|c|}{ 2.4 High intensity vs low-moderate intensity } \\
\hline Jukic 2012 & -57 & 34.1 & 224 & 367 & $10.1 \%$ & $-57.00[-124.00,10.00]$ & & - \\
\hline $\begin{array}{l}\text { Rose } 1991^{\circ} \\
\text { Subtotal }(95 \% \mathrm{Cl})\end{array}$ & -13 & 16.3 & $\begin{array}{r}1281 \\
1505\end{array}$ & $\begin{array}{r}17927 \\
18294\end{array}$ & $\begin{array}{l}12.3 \% \\
22.4 \%\end{array}$ & $\begin{array}{l}-13.00[-44.95,18.95] \\
-24.74[-62.88,13.40]\end{array}$ & & 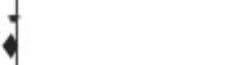 \\
\hline \multicolumn{9}{|c|}{$\begin{array}{l}\text { Heterogeneity: } \mathrm{Tau}^{2}=250.87 ; \mathrm{Ch}^{2}=1.35, \mathrm{df}=1(\mathrm{P}=0.25) ; \mathrm{I}^{2}=26 \% \\
\text { Test for overall effect: } Z=1.27(\mathrm{P}=0.20)\end{array}$} \\
\hline Total $(95 \% \mathrm{Cl})$ & & & 5860 & 79658 & $100.0 \%$ & $-69.85[-114.75,-24.96]$ & & \\
\hline \multicolumn{7}{|c|}{$\begin{array}{l}\text { Heterogeneity: } \text { Tau }^{2}=4006.44 ; \mathrm{Ch}^{2}=80.59, \mathrm{df}=14(\mathrm{P}<0.00001) ; \mathrm{I}^{2}=83 \% \\
\text { Test for overall effect: } Z=3.05(P=0.002) \\
\text { Test for subgroup differences: } \mathrm{Ch}^{2}=17.10, \mathrm{df}=3(\mathrm{P}=0.0007), \mathrm{I}^{2}=82.5 \%\end{array}$} & $\begin{array}{l}1 \\
-1000 \quad-500 \\
\text { PA decreases BV }\end{array}$ & $500 \quad 1000$ \\
\hline
\end{tabular}

Fig. 2 Association between maternal physical activity during pregnancy and infant's birth weight-high levels of physical activity. (2.1) Results from studies providing crude results only, based on activity volume. (2.2) Results from studies providing adjusted results, based on activity volume. (2.3) Results from studies based on duration. (2.4) Results from studies based on intensity. ${ }^{\text {a }}$ Significant difference between groups (nonsignificant here due to estimated equal sample size per quartile). ${ }^{\mathrm{b}}$ This study reported only time spent swimming. ${ }^{\mathrm{C}}$ This study compared highintensity exercise with moderate-intensity exercise. BW, birth weight; PA, physical activity.

the third trimester ( $p$ for linear trend $=0.04$ ). A significant $41.1 \mathrm{~g}$ difference between neonates of women in the highest versus the lowest quartile of total activity was observed after adjustment for various confounders. However, early and midpregnancy physical activity had no significant effect on body composition, nor did meeting exercise recommendations in either trimester.

\section{Association between Maternal Physical Activity and Risk of Low-Birth-Weight and Small for Gestational Age Neonates}

The association between physical activity and LBW was explored in 17 (31.5\%) of the 54 included studies, ${ }^{29-33,35,37,40,42,43,60-62,65,69,71,76}$ including all 4 case-control studies. ${ }^{29-32}$ Adjusted results were presented in 11 of the studies (64.7\%), $29-32,35,40,42,60,61,65,76$ and all but two ${ }^{61,76}$ reported leisure time activity. Eleven of the 13 cohort studies (84.6\%) did not find an association between maternal activity and risk of LBW, ${ }^{33,35,37,40,43,60,62,65,69,71,76}$ while 1 found an increased risk of $\mathrm{LBW}^{61}$ with increasing total activity, and another 1 found a decreased risk of LBW and very $\mathrm{LBW}^{42}$ with regular maternal exercise. As for the case-control studies, two suggested an increased risk of LBW with higher levels of maternal exercise, ${ }^{29,32}$ although one of them also found that low levels of exercise increased the risk of LBW, compared with moderate levels. ${ }^{29}$ The other case-control studies found a decreased risk of LBW with increasing maternal leisure activity. ${ }^{30,31}$

The association between maternal activity and SGA risk was reported in 10 studies $(18.5 \%$ of all studies). ${ }^{44,47,55,57,58,63,66,68,73,75}$ Six of them reported total physical activity $44,55,58,63,73,75$ and six adjusted for potential confounders. ${ }^{47,58,63,66,73,75}$ Overall, most studies (60\%) did not find a significant association between maternal activity and SGA risk. ${ }^{44,47,55,58,68,73}$ However, two studies found an increased risk of SGA with the highest level of either total ${ }^{75}$ or exercise activity ${ }^{57}$ compared with the lowest level, and one study found a decreased risk in women doing exercise compared with those not doing so in the second half of pregnancy. ${ }^{66}$ Finally, one study found that a higher level of total activity in midpregnancy was associated with a decreased risk of SGA, while a higher level of sports and exercise during the same period was associated with an increased risk of SGA. ${ }^{63}$ 


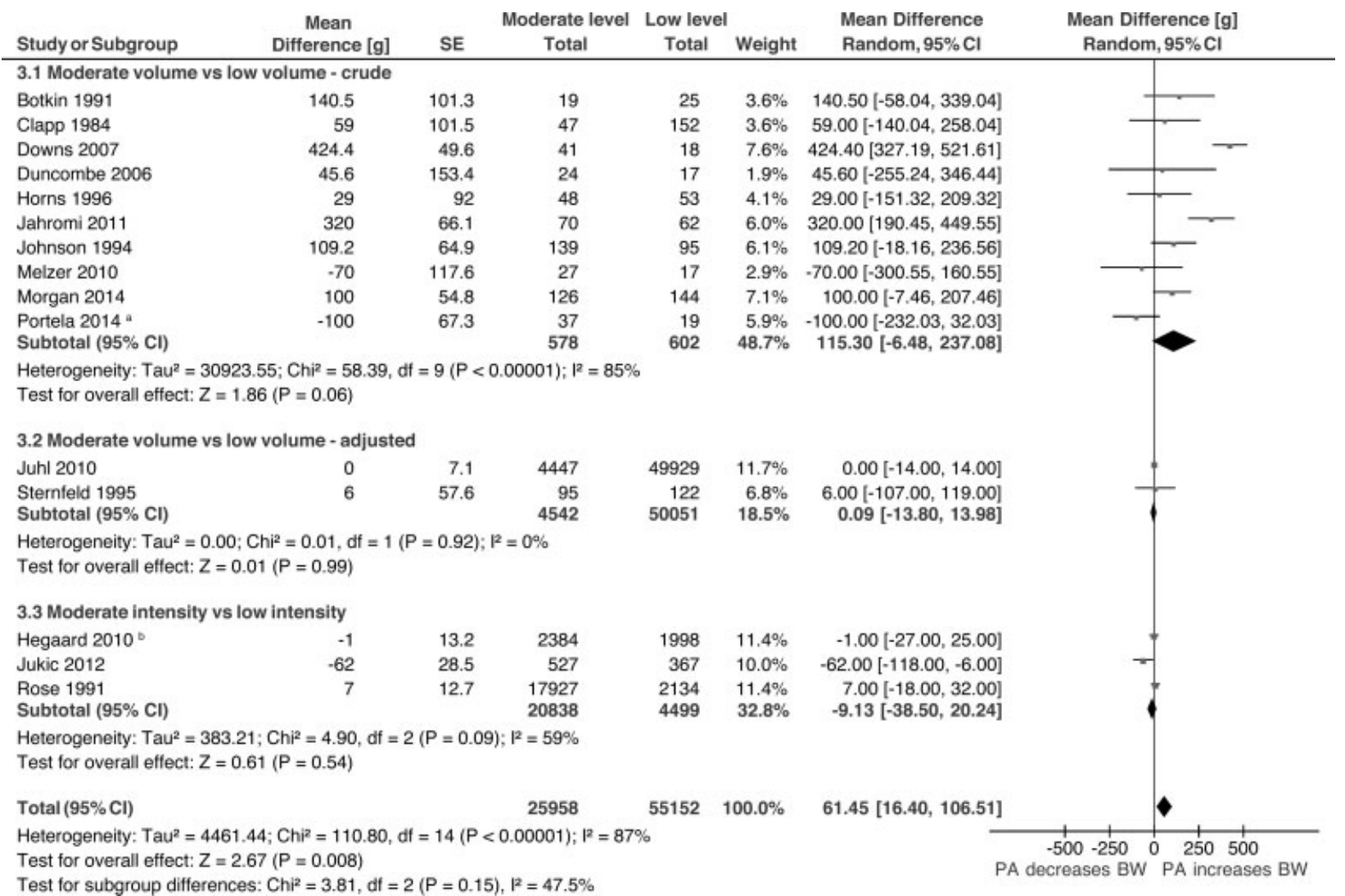

Fig. 3 Association between maternal physical activity during pregnancy and infant's birth weight-moderate levels of physical activity. (3.1) Results from studies providing crude results only, based on activity volume. (3.2) Results from studies providing adjusted results, based on activity volume. (3.3) Results from studies based on intensity. ${ }^{\mathrm{a} T h i s ~ s t u d y ~ m e n t i o n e d ~ n o n s i g n i f i c a n t ~ a d j u s t e d ~ r e s u l t s ~ w i t h o u t ~ p r o v i d i n g ~ e s t i m a t e s . ~}{ }^{\mathrm{b}}$ This study compared light intensity activity with no activity. BW, birth weight; PA, physical activity.

\section{Association between Maternal Physical Activity and Risk of High-Birth-Weight and Large for Gestational Age Neonates}

A total of five studies (9.3\%) reported on HBW, ${ }^{51,62,65,76,77}$ with three of them reporting leisure time activity. $51,62,65$ Three studies found a decreased risk of HBW with increasing total $^{76,77}$ or leisure activity, ${ }^{62}$ the remainder finding no association. ${ }^{51,65}$ From the eight studies reporting on LGA (14.8\%), ${ }^{47,49,55,57,58,66,70,73}$ five reported leisure time physical activity. ${ }^{47,49,57,66,70}$ Four of these studies found a reduced risk of LGA with increasing leisure time activity, ${ }^{47,49,66,70}$ and the fifth one suggested a similar trend (no LGA in the most active group vs. 11 and $17 \%$ of LGA in the other groups). ${ }^{57}$ However, two of these studies showed significant results only in a subsample of women (e.g., nulliparous women ${ }^{70}$ or low-risk women ${ }^{47}$ ). One of the three studies reporting total activity found a decreased risk of LGA with increasing activity, ${ }^{58}$ while the other two found no significant association with risk of LGA. ${ }^{55,73}$

\section{Discussion}

The present systematic review identified 54 observational studies evaluating the association between maternal physical activity during pregnancy and birth weight, the risk of LBW or HBW, risk of SGA or LGA, or body composition. Despite the substantial heterogeneity, results from these studies suggest that moderate levels of physical activity during pregnancy are associated with an increased birth weight, as compared with lower levels. On the contrary, high levels of physical activity were associated with a decreased birth weight. These associations remained when leisure time physical activity alone was considered. Despite the fact that only a few studies evaluated physical activity using objective methods, results from these studies suggested similar conclusions.

Consistent with the decrease in birth weight observed with high physical activity levels, the two studies evaluating neonatal body composition found that high levels of activity were associated with a decreased infant fat mass. Reduced neonatal fat mass was previously found in an RCT comparing women pursuing either a moderate or high volume exercise regimen from the 8th week to end of pregnancy, compared with women pursuing a low volume exercise regimen. ${ }^{19}$ The same authors found no difference in neonatal fat mass or percentage in previously inactive women randomized to either a low volume exercise regimen or no exercise throughout pregnancy, ${ }^{18}$ which is also consistent with the findings of the present review. These observations could either imply that the association between maternal activity and birth weight follows an inverted u-shaped curve, or that a certain amount of exercise is needed before birth weight is reduced. Some observational studies are more suggestive of a dose- 
response relationship; Harrod et $\mathrm{al}^{75}$ showed that the association between total physical activity in late pregnancy and neonatal fat mass followed a linear trend. Another large cohort study ( $n=43,705)$ found that each additional monthly exercise session was associated with a significant $2.1 \mathrm{~g}$ decrease in infant birth weight. ${ }^{62}$

Overall, despite substantial heterogeneity between studies, maternal physical activity does not seem to negatively influence the risk of birth weight extremes. Indeed, the present review highlighted that most studies evaluating HBW and LGA risk found a protective effect with increasing maternal activity, which is in line with the decreased neonatal fat mass or body weight observed in numerous studies. The association between maternal activity and risk of LBW and SGA was less clear, although available studies are reassuring. Globally, results from observational studies are confirmed by a recent meta-analysis evaluating the effects of 28 physical activity interventions during pregnancy. This metaanalysis found a significant reduction in birth weight $(n=5,214)$ and a reduction in the risk of delivering a macrosomic infant ( 19 studies, $n=3,982$ ) without an increased risk of LBW/SGA (11 studies, $n=2,183$ ), with exercise interventions. ${ }^{15}$ However, the impact of exercise dose was not examined in this meta-analysis.

Physical activity is thought to modulate fetal growth through oxygen and nutrient availability and delivery to the fetus. ${ }^{83}$ Indeed, glucose availability is reduced during exercise due to an increased uptake by active muscles, ${ }^{83}$ and uteroplacental blood flow also appears to be decreased with exercise. ${ }^{84,85}$ It has been hypothesized that in response to this intermittent reduction in oxygen and nutrient delivery during exercise, which seems modulated by exercise intensity and/or volume, ${ }^{84-86}$ blood flow is then enhanced at rest. ${ }^{83}$ Consistent with our thorough review, Clapp previously reported the results of a few studies suggesting that a low-tomoderate volume of exercise throughout pregnancy was associated with an increased birth weight while a highintensity, high-volume exercise regimen throughout pregnancy was associated with a decreased birth weight, with various effects in between. ${ }^{83}$ Accordingly, it appears that below a certain threshold, the reduced nutrient availability induced by exercise is compensated and results in enhanced fetal growth, while above this threshold nutrient availability cannot be completely compensated, leading to reduced birth weight. Of course, glucose availability and delivery to the fetus is also influenced by other factors, including maternal nutrition, health condition, and fitness, ${ }^{83,87}$ thus it is possible that the effects of exercise on fetal growth are seldom counterbalanced by other maternal behaviors and conditions. Thus, this suggested inverted $\mathrm{U}$-shape relationship between physical activity and birth weight, which can be modulated by factors such as maternal diet, could explain the discrepancy observed in RCTs' results.

Previous meta-analyses of RCTs also showed a small reduction in infant's birth weight with physical activity interventions, but were unable to distinguish the effects of various volumes or intensity of physical activity. ${ }^{15,16} \mathrm{Al}-$ though it was not possible to combine the results in a quantitative estimate for all outcomes and comparisons, we still attempted to determine the influence of physical activity volume on birth weight. Despite significant statistical heterogeneity, our prudent classification of studies provided new insights on the association between maternal physical activity and birth weight. However, whether our classification was adequate to truly discriminate moderate level from high level of physical activity remains debatable, as we mostly relied on the studies' own classification of physical activity levels. For instance, although some studies stated having evaluated vigorous intensity activity, their definition of vigorous intensity did not necessarily comply with accepted definitions ( $\geq 60 \%$ aerobic capacity reserve) ${ }^{88}$ or was somewhat imprecise. This implies that the measure of physical activity in these studies likely included both moderate and vigorous intensity activity. Thus, adequately defining intensity will be of prime importance in future studies, to accurately evaluate the impact of various physical activity intensities on pregnancy outcomes.

\section{Limitations}

This systematic review included observational studies evaluating the association between maternal physical activity and birth weight outcomes; therefore, the associations observed may not constitute causal relationships. Results must also be interpreted with caution due to the high heterogeneity found between study results, methodology, and quality, which on one hand limited the possibility to pool some studies in the meta-analysis, leading to a potential selection bias, and on the other hand might also have decreased the strength of the associations found in the present review. It should also be acknowledged that study results were included in the analyses, even when adjustment for confounders was lacking. Indeed, more than one-third of the studies included in the present review did not adjust for covariates known or thought to influence physical activity and fetal growth. Finally, publication bias cannot be ruled out, as some studies with null results might not have been published. Although a publication bias would imply that the associations observed in the present review overestimate the real association between maternal physical activity and birth weight, and despite the aforementioned limitations, we believe that it is unlikely that our finding of a different association between maternal physical activity and birth weight depending on physical activity volume would be discredited. Nevertheless, further confirmation of this volume-dependent association is needed.

\section{Recommendations for Future Research}

The present review highlighted that observational studies can help us gain a better insight of the mechanisms explaining the association between maternal activity and birth weight outcomes. However, to further clarify the association between maternal physical activity and birth weight, some issues need to be addressed first. For instance, a precise and meticulous measure of maternal physical activity, including all physical activity parameters, will be needed to identify the potential thresholds where physical activity can be expected to 
influence birth weight differently. As previously proposed, since physical activity might exert only a modest effect on birth weight outcomes, accurate methods to measure physical activity are needed to avoid missing this effect because of measurement error. ${ }^{89}$ Accordingly, studies using objective measurement methods such as accelerometry, which are thought to provide more precise and accurate estimates of physical activity, free of recall, and self-report bias, are needed as only five studies using accelerometry were available for inclusion in the present review. However, efforts should also be made in harmonizing accelerometry data processing in pregnancy studies to allow a better reliability of physical activity estimates and comparability between studies, since no cut point has been developed specifically for pregnancy and no consensus has been made regarding data processing, leading to great methodological variability regarding accelerometry data in pregnancy studies. ${ }^{73,77,90,91}$

Also, although the decreased birth weight observed with increasing physical activity in the present review and in previous meta-analyses of RCTs might be beneficial for the child's health, it is possible that this effect could become detrimental above a certain level of physical activity, leading to an increased risk of SGA or other adverse outcomes. Obviously, the identification of this potential threshold will not be possible through RCTs for ethical reasons. The same situation applies regarding the effects of vigorous intensity activity on pregnancy outcomes, or the effects of physical activity in high-risk populations, emphasizing the need for additional high-quality observational studies. Similarly, to improve our understanding of the impact of physical activity performed during the first trimester, which remains limited due to the difficulty in initiating a physical activity intervention before the end of this trimester, observational studies appear as an important alternative, complementing knowledge acquired through RCTs. To further precise the association between physical activity and fetal growth, future research should also focus on outcomes such as neonatal body composition and mechanistic pathways while considering important confounders.

Refining physical activity guidelines for pregnant women to optimize fetal growth will only be possible when the aforementioned issues and others will be adequately answered. In light of available evidence, we believe that this can only be achieved through high-quality observational studies and RCTs.

\section{Conclusion}

Based on observational studies, maternal physical activity during pregnancy does not seem to negatively influence birth weight or risk of birth weight extremes in healthy, low-risk pregnant women. The association between maternal physical activity levels and infant's birth weight suggests an inverted u-shaped curve, where moderate levels seem associated with an increased birth weight while high levels of physical activity seem associated with a decreased birth weight. Maternal physical activity also appears to be associated with a reduced neonatal fat mass, but the small number of studies evaluating neonatal body composition precludes definitive conclusions. Future studies should focus on precisely defining and measuring physical activity in each trimester to determine the volume, type, and intensity of physical activity needed to influence the neonate's body size and composition and to allow better study comparability. Underrepresented groups of pregnant women, such as women with high-risk pregnancy, should also be studied. As the intrauterine environment might have a long term influence on the child's health, it is crucial to evaluate the effects of various levels and patterns of physical activity during pregnancy to identify the optimal physical activity prescription. A careful monitoring of physical activity type, frequency, intensity, and duration in each trimester of pregnancy and of its effects on pregnancy outcomes in various populations will definitely contribute to this important objective.

\section{Acknowledgments}

The authors thank William Witteman, information specialist at the CHU de Québec, who performed the electronic database search. This study was funded by the Fondation des Étoiles and authors supported by awards from the Canadian Institutes of Health Research and the Fond de Recherche du Québec-Santé.

\section{Note}

Protocol Registration Prospero, available at: http://www. crd.york.ac.uk/PROSPERO/, CRD42013004968.

\section{References}

1 Singhal A, Wells J, Cole TJ, Fewtrell M, Lucas A. Programming of lean body mass: a link between birth weight, obesity, and cardiovascular disease? Am J Clin Nutr 2003;77(3):726-730

2 Inskip HM, Godfrey KM, Martin HJ, Simmonds SJ, Cooper C, Sayer AA; Southampton Women's Survey Study Group. Size at birth and its relation to muscle strength in young adult women. J Intern Med 2007;262(3):368-374

3 Ortega FB, Labayen I, Ruiz JR, et al; AVENA Study Group. Are muscular and cardiovascular fitness partially programmed at birth? Role of body composition. J Pediatr 2009;154(1):61-66.e1

4 Ridgway CL, Ong KK, Tammelin T, Sharp SJ, Ekelund U, Jarvelin MR. Birth size, infant weight gain, and motor development influence adult physical performance. Med Sci Sports Exerc 2009;41(6): 1212-1221

5 Labayen I, Moreno LA, Ruiz JR, et al; Avena Study Group. Small birth weight and later body composition and fat distribution in adolescents: the Avena study. Obesity (Silver Spring) 2008;16(7): 1680-1686

6 Chiavaroli V, Giannini C, D’Adamo E, de Giorgis T, Chiarelli F, Mohn A. Insulin resistance and oxidative stress in children born small and large for gestational age. Pediatrics 2009;124(2): 695-702

7 Stuart A, Amer-Wåhlin I, Persson J, Källen K. Long-term cardiovascular risk in relation to birth weight and exposure to maternal diabetes mellitus. Int J Cardiol 2013;168(3):2653-2657

8 Shi Y, De Groh M, Morrison H. Perinatal and early childhood factors for overweight and obesity in young Canadian children. Can J Public Health 2013;104(1):e69-e74 
9 Kramer MS, McDonald SW. Aerobic exercise for women during pregnancy. Cochrane Database Syst Rev 2006;(3):CD000180

10 Thangaratinam S, Rogozińska E, Jolly K, et al. Interventions to reduce or prevent obesity in pregnant women: a systematic review. Health Technol Assess 2012;16(31):iii-iv, 1-191

11 Aune D, Saugstad OD, Henriksen T, Tonstad S. Physical activity and the risk of preeclampsia: a systematic review and meta-analysis. Epidemiology 2014;25(3):331-343

12 Domenjoz I, Kayser B, Boulvain M. Effect of physical activity during pregnancy on mode of delivery. Am J Obstet Gynecol 2014;211(4): 401.e1-401.e11

13 Russo LM, Nobles C, Ertel KA, Chasan-Taber L, Whitcomb BW. Physical activity interventions in pregnancy and risk of gestational diabetes mellitus: a systematic review and meta-analysis. Obstet Gynecol 2015;125(3):576-582

14 Sanabria-Martínez G, García-Hermoso A, Poyatos-León R, ÁlvarezBueno C, Sánchez-López M, Martínez-Vizcaíno V. Effectiveness of physical activity interventions on preventing gestational diabetes mellitus and excessive maternal weight gain: a meta-analysis. BJOG 2015;122(9):1167-1174

15 Wiebe HW, Boulé NG, Chari R, Davenport MH. The effect of supervised prenatal exercise on fetal growth: a meta-analysis. Obstet Gynecol 2015;125(5):1185-1194

16 Sanabria-Martínez G, García-Hermoso A, Poyatos-León R, González-García A, Sánchez-López M, Martínez-Vizcaíno V. Effects of exercise-based interventions on neonatal outcomes: A metaanalysis of randomized controlled trials. Am J Health Promot 2015 (e-pub ahead of print). doi: 10.4278/ajhp.140718-LIT-351

17 de Oliveria Melo AS, Silva JL, Tavares JS, Barros VO, Leite DF, Amorim MM. Effect of a physical exercise program during pregnancy on uteroplacental and fetal blood flow and fetal growth: a randomized controlled trial. Obstet Gynecol 2012;120(2 Pt 1):302-310

18 Clapp JF III, Kim H, Burciu B, Lopez B. Beginning regular exercise in early pregnancy: effect on fetoplacental growth. Am J Obstet Gynecol 2000;183(6):1484-1488

19 Clapp JF III, Kim H, Burciu B, Schmidt S, Petry K, Lopez B. Continuing regular exercise during pregnancy: effect of exercise volume on fetoplacental growth. Am J Obstet Gynecol 2002; 186(1):142-147

20 Jackson MR, Gott P, Lye SJ, Ritchie JW, Clapp JF III. The effects of maternal aerobic exercise on human placental development: placental volumetric composition and surface areas. Placenta 1995;16(2):179-191

21 Davies GA, Wolfe LA, Mottola MF, MacKinnon C; Society of Obstetricians and gynecologists of Canada, SOGC Clinical Practice Obstetrics Committee. Joint SOGC/CSEP clinical practice guideline: exercise in pregnancy and the postpartum period. Can J Appl Physiol 2003;28(3):330-341

22 ACOG Committee Obstetric Practice. ACOG Committee opinion. Number 267, January 2002: exercise during pregnancy and the postpartum period. Obstet Gynecol 2002;99(1):171-173

23 Wells GA, Shea B, O'Connell D, et al. The Newcastle-Ottawa Scale (NOS) for assessing the quality of nonrandomised studies in metaanalyses. Available at: http://www.ohri.ca/programs/clinical_epidemiology/oxford.asp. Accessed January 28, 2014

24 Bisson M, Alméras N, Plaisance J, et al. Maternal fitness at the onset of the second trimester of pregnancy: correlates and relationship with infant birth weight. Pediatr Obes 2013;8(6):464-474

25 Lynch KE, Landsbaugh JR, Whitcomb BW, Pekow P, Markenson G, Chasan-Taber L. Physical activity of pregnant Hispanic women. Am J Prev Med 2012;43(4):434-439

26 Haskell WL, Lee IM, Pate RR, et al; American College of Sports Medicine; American Heart Association. Physical activity and public health: updated recommendation for adults from the American College of Sports Medicine and the American Heart Association. Circulation 2007;116(9):1081-1093
27 DerSimonian R, Laird N. Meta-analysis in clinical trials. Control Clin Trials 1986;7(3):177-188

28 Higgins JP, Thompson SG, Deeks JJ, Altman DG. Measuring inconsistency in meta-analyses. BMJ 2003;327(7414):557-560

29 Campbell MK, Mottola MF. Recreational exercise and occupational activity during pregnancy and birth weight: a case-control study. Am J Obstet Gynecol 2001;184(3):403-408

30 Schramm WF, Stockbauer JW, Hoffman HJ. Exercise, employment, other daily activities, and adverse pregnancy outcomes. Am J Epidemiol 1996;143(3):211-218

31 Takito MY, Benício MH. Physical activity during pregnancy and fetal outcomes: a case-control study. Rev Saude Publica 2010; 44(1):90-101

32 Mahmoodi Z, Karimlou M, Sajjadi H, et al. Physical Activity Pattern and Personal-Social Factors of Mothers During Pregnancy And Infant Birth Weight Based On MET Scale: A Case-Control Study. Iran Red Crescent Med J 2013;15(7):573-580

33 Bell RJ, Palma SM, Lumley JM. The effect of vigorous exercise during pregnancy on birth-weight. Aust N Z J Obstet Gynaecol 1995;35(1):46-51

34 Botkin C, Driscoll CE. Maternal aerobic exercise: newborn effects. Fam Pract Res J 1991;11(4):387-393

35 Cavalli AS, Tanaka T. Maternal leisure-time physical activities are not determinant risk factors of low birthweight babies: A crosssectional study of 1,714 pregnant women. Environ Health Prev Med 2000;5(2):72-80

36 Clapp JF 3rd, Capeless EL. Neonatal morphometrics after endurance exercise during pregnancy. Am J Obstet Gynecol 1990;163(6 Pt 1):1805-1811

37 Duncombe D, Skouteris H, Wertheim EH, Kelly L, Fraser V, Paxton SJ. Vigorous exercise and birth outcomes in a sample of recreational exercisers: a prospective study across pregnancy. Aust N Z J Obstet Gynaecol 2006;46(4):288-292

38 Koushkie Jahromi M, Namavar Jahromi B, Hojjati S. Relationship between daily physical activity during last month of pregnancy and pregnancy outcome. Iran Red Crescent Med J 2011;13(1): $15-20$

39 Johnson AA, Knight EM, Edwards $\mathrm{CH}$, et al. Selected lifestyle practices in urban African American women-relationships to pregnancy outcome, dietary intakes and anthropometric measurements. J Nutr 1994;124(6, Suppl):963S-972S

40 Klebanoff MA, Shiono PH, Carey JC. The effect of physical activity during pregnancy on preterm delivery and birth weight. Am J Obstet Gynecol 1990;163(5 Pt 1):1450-1456

41 Langhoff-Roos J, Lindmark G, Kylberg E, Gebre-Medhin M. Energy intake and physical activity during pregnancy in relation to maternal fat accretion and infant birthweight. Br J Obstet Gynaecol 1987;94(12):1178-1185

42 Leiferman JA, Evenson KR. The effect of regular leisure physical activity on birth outcomes. Matern Child Health J 2003;7(1):59-64

43 Lobel M, Cannella DL, Graham JE, DeVincent C, Schneider J, Meyer BA. Pregnancy-specific stress, prenatal health behaviors, and birth outcomes. Health Psychol 2008;27(5):604-615

44 Magann EF, Evans SF, Newnham JP. Employment, exertion, and pregnancy outcome: assessment by kilocalories expended each day. Am J Obstet Gynecol 1996;175(1):182-187

45 Melzer K, Schutz Y, Soehnchen N, et al. Effects of recommended levels of physical activity on pregnancy outcomes. Am J Obstet Gynecol 2010;202(3):266.e1-266.e6

46 Montpetit AE, Plourde H, Cohen TR, Koski KG. Modeling the impact of prepregnancy BMI, physical activity, and energy intake on gestational weight gain, infant birth weight, and postpartum weight retention. J Phys Act Health 2012;9(7):1020-1029

47 Mudd LM, Pivarnik J, Holzman CB, Paneth N, Pfeiffer K, Chung H. Leisure-time physical activity in pregnancy and the birth weight distribution: where is the effect? J Phys Act Health 2012;9(8): 1168-1177 
48 Perkins CC, Pivarnik JM, Paneth N, Stein AD. Physical activity and fetal growth during pregnancy. Obstet Gynecol 2007;109(1): 81-87

49 Snapp CA, Donaldson SK. Gestational diabetes mellitus: physical exercise and health outcomes. Biol Res Nurs 2008;10(2): 145-155

50 Sternfeld B, Quesenberry CP Jr, Eskenazi B, Newman LA. Exercise during pregnancy and pregnancy outcome. Med Sci Sports Exerc 1995;27(5):634-640

51 Voldner N, Frøslie KF, Bo K, et al. Modifiable determinants of fetal macrosomia: role of lifestyle-related factors. Acta Obstet Gynecol Scand 2008;87(4):423-429

52 Watson PE, McDonald BW. Activity levels in pregnant New Zealand women: relationship with socioeconomic factors, well-being, anthropometric measures, and birth outcome. Appl Physiol Nutr Metab 2007;32(4):733-742

53 Zeanah M, Schlosser SP. Adherence to ACOG guidelines on exercise during pregnancy: effect on pregnancy outcome. J Obstet Gynecol Neonatal Nurs 1993;22(4):329-335

54 Mäkelä J, Lagström H, Kaljonen A, Simell O, Niinikoski H. Hyperglycemia and lower diet quality in pregnant overweight women and increased infant size at birth and at 13 months of age-STEPS study. Early Hum Dev 2013;89(6):439-444

55 Portela SN, Rocha-de-Souza R, Oppermann-Lisboa K, Donatto GB, Dal Bosco SN, El Beitune P. Maternal physical activity, cervical length and its relation to spontaneous vaginal birth at term. Arch Gynecol Obstet 2014;290(2):257-262

56 Rice PL, Fort IL. The relationship of maternal exercise on labor, delivery and health of the newborn. J Sports Med Phys Fitness 1991;31(1):95-99

57 Clapp JF III, Dickstein S. Endurance exercise and pregnancy outcome. Med Sci Sports Exerc 1984;16(6):556-562

58 Alderman BW, Zhao H, Holt VL, Watts DH, Beresford SA. Maternal physical activity in pregnancy and infant size for gestational age. Ann Epidemiol 1998;8(8):513-519

59 Both MI, Overvest MA, Wildhagen MF, Golding J, Wildschut HIJ. The association of daily physical activity and birth outcome: a population-based cohort study. Eur J Epidemiol 2010;25(6): 421-429

60 Dumith SC, Domingues MR, Mendoza-Sassi RA, Cesar JA. Physical activity during pregnancy and its association with maternal and child health indicators. Rev Saude Publica 2012;46(2): 327-333

61 Dwarkanath P, Muthayya S, Vaz M, et al. The relationship between maternal physical activity during pregnancy and birth weight Asia Pac J Clin Nutr 2007;16(4):704-710

62 Fleten C, Stigum H, Magnus P, Nystad W. Exercise during pregnancy, maternal prepregnancy body mass index, and birth weight. Obstet Gynecol 2010;115(2 Pt 1):331-337

63 Gollenberg AL, Pekow P, Bertone-Johnson ER, Freedson PS, Markenson G, Chasan-Taber L. Physical activity and risk of small-forgestational-age birth among predominantly Puerto Rican women. Matern Child Health J 2011;15(1):49-59

64 Hatch MC, Shu XO, McLean DE, et al. Maternal exercise during pregnancy, physical fitness, and fetal growth. Am J Epidemiol 1993;137(10):1105-1114

65 Hegaard HK, Petersson K, Hedegaard M, et al. Sports and leisuretime physical activity in pregnancy and birth weight: a population-based study. Scand J Med Sci Sports 2010;20(1):e96-e102

66 Juhl M, Olsen J, Andersen PK, Nøhr EA, Andersen AM. Physical exercise during pregnancy and fetal growth measures: a study within the Danish National Birth Cohort. Am J Obstet Gynecol 2010;202(1):63.e1-63.e8

67 Jukic AM, Evenson KR, Daniels JL, Herring AH, Wilcox AJ, Hartmann KE. A prospective study of the association between vigorous physical activity during pregnancy and length of gestation and birthweight. Matern Child Health J 2012;16(5):1031-1044
68 Magann EF, Evans SF, Weitz B, Newnham J. Antepartum, intrapartum, and neonatal significance of exercise on healthy, low-risk pregnant women. Obstet Gynecol 2002; 99(3):466-472

69 Orr ST, James SA, Garry J, Prince CB, Newton ER. Exercise and pregnancy outcome among urban, low-income, black women. Ethn Dis 2006;16(4):933-937

70 Owe KM, Nystad W, Bø K. Association between regular exercise and excessive newborn birth weight. Obstet Gynecol 2009;114(4): 770-776

71 Rose NC, Haddow JE, Palomaki GE, Knight GJ. Self-rated physical activity level during the second trimester and pregnancy outcome. Obstet Gynecol 1991;78(6):1078-1080

72 Wojtyła A, Kapka-Skrzypczak L, Paprzycki P, Skrzypczak M, Biliński P. Epidemiological studies in Poland on effect of physical activity of pregnant women on the health of offspring and future generations - adaptation of the hypothesis development origin of health and diseases. Ann Agric Environ Med 2012; 19(2):315-326

73 Morgan KL, Rahman MA, Hill RA, et al. Physical activity and excess weight in pregnancy have independent and unique effects on delivery and perinatal outcomes. PLoS ONE 2014;9(4):e94532

74 Nieuwenhuijsen MJ, Northstone K, Golding J; ALSPAC Study Team. Swimming and birth weight. Epidemiology 2002;13(6): 725-728

75 Harrod CS, Chasan-Taber L, Reynolds RM, et al. Physical activity in pregnancy and neonatal body composition: the Healthy Start study. Obstet Gynecol 2014;124(2 Pt 1):257-264

76 Currie LM, Woolcott CG, Fell DB, Armson BA, Dodds L. The association between physical activity and maternal and neonatal outcomes: a prospective cohort. Matern Child Health J 2014;18(8): 1823-1830

77 Reid EW, McNeill JA, Alderdice FA, Tully MA, Holmes VA. Physical activity, sedentary behaviour and fetal macrosomia in uncomplicated pregnancies: a prospective cohort study. Midwifery 2014; 30(12):1202-1209

78 Dale E, Mullinax KM, Bryan DH. Exercise during pregnancy: effects on the fetus. Can J Appl Sport Sci 1982;7(2):98-103

79 Downs DS, Hausenblas HA. Pregnant women's third trimester exercise behaviors, body mass index, and pregnancy outcomes. Psychol Health 2007;22(5):545-559

80 Horns PN, Ratcliffe LP, Leggett JC, Swanson MS. Pregnancy outcomes among active and sedentary primiparous women. J Obstet Gynecol Neonatal Nurs 1996;25(1):49-54

81 Jarrett JC II, Spellacy WN. Jogging during pregnancy: an improved outcome? Obstet Gynecol 1983;61(6):705-709

82 Mparmpakas D, Goumenou A, Zachariades E, Pados G, Gidron Y, Karteris E. Immune system function, stress, exercise and nutrition profile can affect pregnancy outcome: Lessons from a Mediterranean cohort. Exp Ther Med 2013;5(2):411-418

83 Clapp JF. Influence of endurance exercise and diet on human placental development and fetal growth. Placenta 2006;27(6-7): 527-534

84 Erkkola RU, Pirhonen JP, Kivijärvi AK. Flow velocity waveforms in uterine and umbilical arteries during submaximal bicycle exercise in normal pregnancy. Obstet Gynecol 1992;79(4): 611-615

85 Salvesen KA, Hem E, Sundgot-Borgen J. Fetal wellbeing may be compromised during strenuous exercise among pregnant elite athletes. Br J Sports Med 2012;46(4):279-283

86 Ruchat SM, Davenport MH, Giroux I, et al. Effect of exercise intensity and duration on capillary glucose responses in pregnant women at low and high risk for gestational diabetes. Diabetes Metab Res Rev 2012;28(8):669-678

87 McGowan CA, McAuliffe FM. The influence of maternal glycaemia and dietary glycaemic index on pregnancy outcome in healthy mothers. Br J Nutr 2010;104(2):153-159 
88 Physical Activity Guidelines for Americans. Washington D.C.: U.S. Department of Health and Human Services; 2008:76. Available at: http://www.health.gov/PAGuidelines. Accessed October 28, 2015

89 Chasan-Taber L, Evenson KR, Sternfeld B, Kengeri S. Assessment of recreational physical activity during pregnancy in epidemiologic studies of birthweight and length of gestation: methodologic aspects. Women Health 2007;45(4):85-107
90 Evenson KR, Wen F. Prevalence and correlates of objectively measured physical activity and sedentary behavior among US pregnant women. Prev Med 2011;53(1-2):39-43

91 Bisson M, Alméras N, Dufresne SS, et al. A 12-Week Exercise Program for Pregnant Women with Obesity to Improve Physical Activity Levels: An Open Randomised Preliminary Study. PLoS ONE 2015;10(9):e0137742 